\title{
Mollusken-Gemeinschaften der Bahía de Nenguange (Karibik, Kolumbien)
}

\author{
J. M. Diaz M. ${ }^{1}$ \& K.-J. Götting ${ }^{2}$ \\ ${ }^{1}$ INVEMAR, Santa Marta, Colombia, S. A. \\ ${ }^{2}$ Institut für Allgemeine und Spezielle Zoologie, \\ Justus-Liebig-Universität Gießen; D-6300 Gießen, Bundesrepublik Deutschland
}

\begin{abstract}
Mollusc communities of the Bahía de Nenguange (Caribbean Sea, Colombia). The Bahía de Nenguange is one of the largest bays $\left(5 \mathrm{~km}^{2}\right)$ east of Santa Marta. The shore of the Ushaped bay is made up of steep slate rocks, interrupted by sandy beaches. The waters within the bay are rather shallow but get deeper to the north; in the western part, which is $10-12 \mathrm{~m}$ deep, a platform is situated. The western shore is subject to strong exposure of waves; the eastern half is calmer. On the eastern beach a small stand of mangroves is located. Altogether, 491 species of molluscs were found, belonging to 100 families and 259 genera, 12 molluscan assemblages have been documented: 4 distributed in the intertidal, and 8 in the sublittoral zone. The distribution of the sublittoral coenoses depends on the distribution of the substrates. Numerous microgastropods occur in coarse-grained sediments. Highest diversity was observed on fine and mixed sands. Variations documented in the diversity of the Thalassia-coenosis and of the fine sand-mixed sand coenoses are due to changes in numbers of species, whereas the diversity on ooze is due likewise to modifications in the number of species and to evenness.
\end{abstract}

\section{EINLEITUNG}

Über die Malakofauna der karibischen Küste Kolumbiens sind seit den ersten Informationen durch Dautzenberg (1900) einige weitere Arbeiten publiziert worden (u. a. Daniel, 1941; Kaufmann \& Götting, 1970; Cosel, 1973; Götting, 1973; Marcus, 1976; Duque, 1979), doch handelt es sich in der Mehrzahl um faunistische Veröffentlichungen, die wenige oder gar keine ökologischen Angaben enthalten. Die benachbarten Küsten von Venezuela und Surinam haben eine artenreiche Malakofauna, wie die Publikationen von Almeida (1974), Altena $(1968,1971,1975)$, Petuch $(1976,1980,1981)$, Princz $(1973,1977,1978,1982)$ und Tello (1975) zeigen. Für eine umfassende Bestandsaufnahme der Molluskenfauna, insbesondere aber zur Aufklärung der ökologischen Beziehungen zwischen den Arten und den Artengemeinschaften der Weichtiere schien die Bahía de Nenguange östlich von Santa Marta gut geeignet, da sie auf überschaubarem Raum eine Vielzahl verschiedener Biotope aufweist.

\section{DAS UNTERSUCHUNGSGEBIET}

Die Bahía de Nenguange liegt im westlichen Teil des Tayrona-Nationalparks bei $11^{\circ} 20^{\prime} \mathrm{N} 74^{\circ} 05^{\prime} \mathrm{W}$ in unmittelbarer Nähe der Sierra Nevada de Santa Marta, die in ca. 50 
$\mathrm{km}$ Entfernung Höhen von rund $5000 \mathrm{~m}$ erreicht. Die Bucht ist mit etwa $5 \mathrm{~km}^{2}$ die größte in dieser Region und mündet mit einer rund $1,5 \mathrm{~km}$ weiten Öffnung im Norden in die Karibische See. Das Küstenrelief innerhalb der Bucht ist durch exponierte Felsabschnitte (phyllitische Schiefer, Grün- bzw. Glimmerschiefer) und unterschiedlich geschützte Strände geprägt. Die zwei größten Strandabschnitte liegen im SW (Playa Brava) und im $\mathrm{S}$ (Playa Oriental) (Abb. 1,2), weitere am O-Ufer. Auf der W-Seite der Bucht finden sich, bis auf die kleine Playa Placelito, steile Felsen. Von Dezember bis März ist das Gebiet den ONO-Passaten ausgesetzt und niederschlagsfrei. Von Juni bis Oktober erstreckt sich die Tropikfront über diesen Küstenbereich, was zu großräumigen Aufwärtsbewegungen der Luft, zu Wolkenbildung und nachfolgenden Regenfällen führt. Der Passateinfluß ist dann zwar schwach, aber bemerkbar. Die Niederschlagswerte liegen zwischen 700 und $900 \mathrm{~mm}$ im Jahr, wovon etwa $1 / 3 \mathrm{im}$ Oktober und November fällt. Die mittlere Lufttemperatur in der Nenguange beträgt $27^{\circ} \mathrm{C}$ (Heybrock, 1984), der Unterschied zwischen dem kältesten (Oktober) und dem wärmsten Monat (August) ist geringfügig (ca. $3^{\circ} \mathrm{C}$ ). Dagegen sind die tageszeitlichen Unterschiede ausgeprägt: Tagestemperaturen von $38^{\circ} \mathrm{C}$ sind ebenso häufig wie Tiefsttemperaturen von $22^{\circ} \mathrm{C}$ in der Morgendämmerung.

Die terrestrische Vegetation besteht aus einem regengrünen Trockenwald, in dem Dornbuschsträucher (u. a. Mimosaceae, Burseraceae) und Kakteen vorherrschen (Heybrock, 1984; Schnetter, 1968; Wilhelmy, 1954). Landeinwärts der Playa Oriental, einem durch jüngere Aufschüttungen verlandeten Flachbereich, gedeiht stellenweise Mangrove (Laguncularia racemosa, Conocarpus spec.). Am östlichen Ufer wächst Mangroven-Buschholz in Kontakt zum Meer. Es besteht aus 4-5 m hohen Rhizophora mangleSträuchern, deren Stelzwurzeln bis in etwa $1 \mathrm{~m}$ Wassertiefe reichen. Dieser, durchschnittlich $4 \mathrm{~m}$ breite Rhizophora-Gürtel wird landein vorwiegend von Laguncularia-

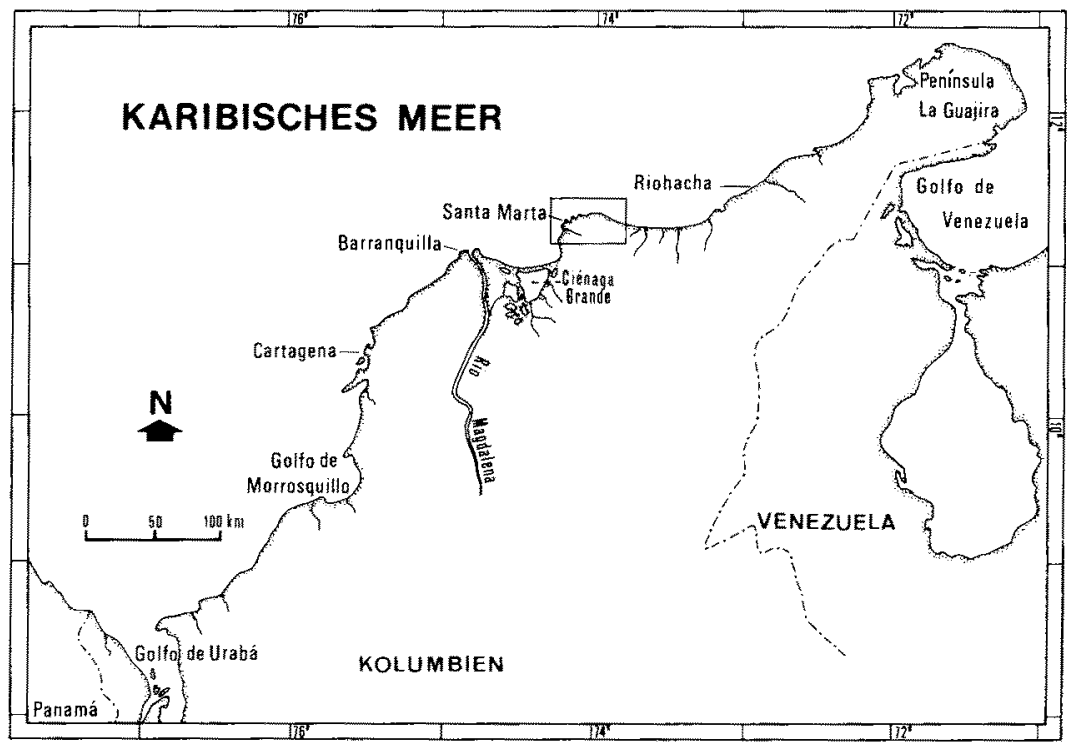

Abb. 1. Die Lage des Untersuchungsgebietes (umrahmt) an der N-Küste Kolumbiens 


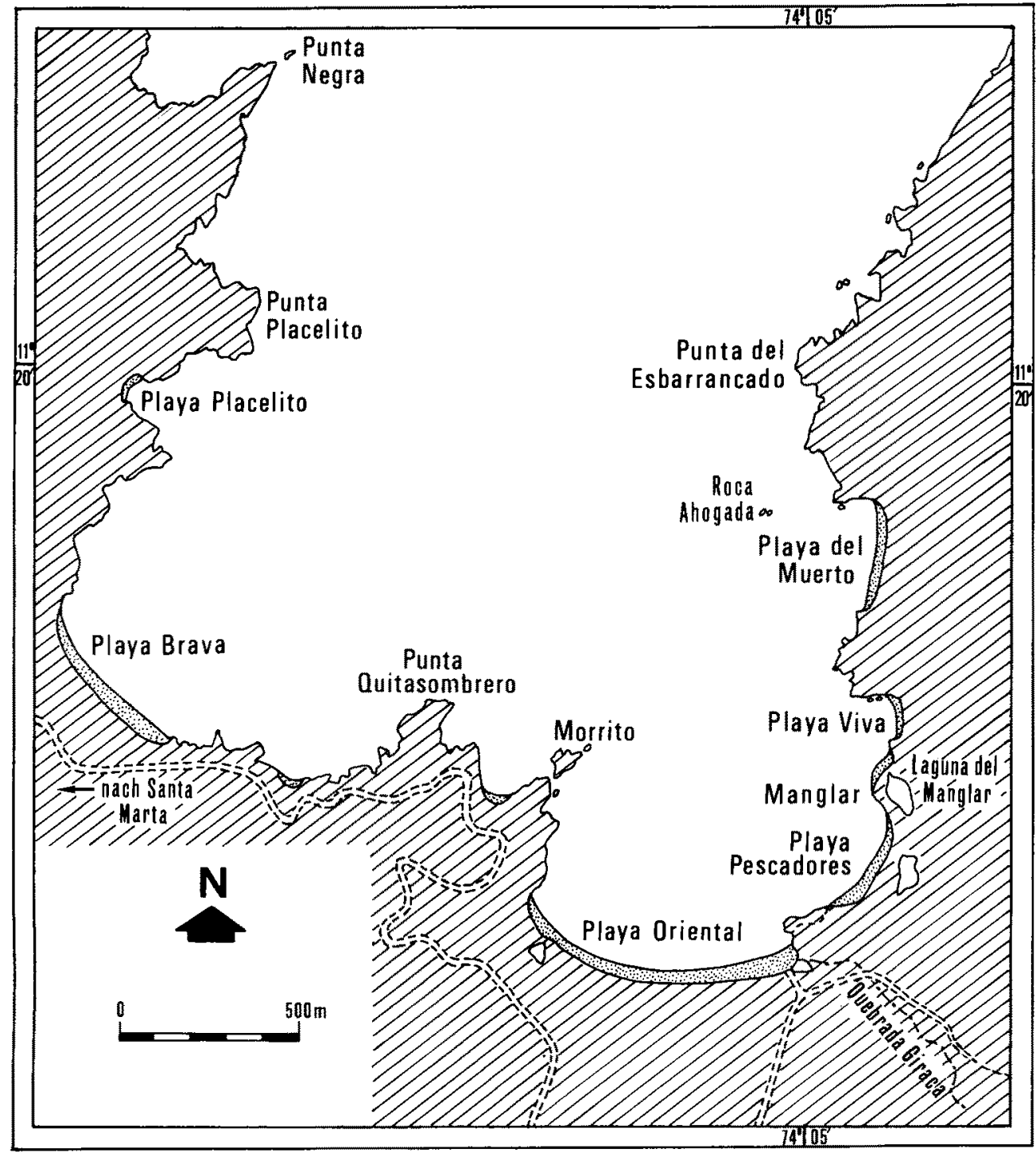

Abb. 2. Die Bahía de Nenguange

und Avicennia-Bäumen ersetzt, die eine lediglich während der Regenzeit mit 30-40 cm tiefem Brackwasser gefüllte Lagune umsäumen.

Ein kleiner Bach, die Quebrada Giraca, bildet die einzige Süßwasserzufuhr. Er mündet in eine Lagune am Ende der Playa Oriental, die nur in niederschlagsreichen Zeiten den Strandwall durchbricht und sich damit zum Meer öffnet. Die Gezeiten sind unregelmäßig durch ein Zusammenwirken von täglichen und halbtäglichen Tidentypen (Brattström, 1980); der Tidenhub beträgt nur etwa $25 \mathrm{~cm}$. Die Oberflächentemperatur des Wassers liegt im Sommer (Mai bis November) bei $28^{\circ} \mathrm{C}$; sie sinkt von Dezember bis 
April auf durchschnittlich $24^{\circ} \mathrm{C}$. Dann können küstenparallel und ablandig wehende Winde die oberflächlichen Wassermassen ins offene Meer treiben, so daß kaltes Tiefenwasser aufsteigen kann (Bula, 1977; Caycedo, 1977; Wedler, 1975). Die Strömungen vor der Küste werden durch den Nordäquatorialstrom bestimmt, der entlang der venezolanischen Küste westwärts in die Karibik vordringt. Einer seiner Ausläufer trifft bei Santa Marta auf den aus SW kommenden Gegenstrom; er dringt von NO in die Nenguange ein und beeinflußt vor allem die Westhälfte der Bucht.

Korallenriffe und Seegraswiesen sind vorwiegend am östlichen Ufer zu finden. Die ausgedehntesten Seegraswiesen (vor allem aus Thalassia testudinum) und die auffäligsten Riff-Formationen sind im Bereich der Playa Pescadores und der Playa Viva anzutreffen. Weitere Korallenriffe erstrecken sich parallel zur Playa del Muerto sowie direkt unterhalb der Felsen entlang des nordöstlichen Ufers, um die Kleininsel Morrito und um eine sich aus 15-20 m Tiefe erhebende, felsige Untiefe (Roca Ahogada). Die einzigen menschlichen Bewohner der Bahía de Nenguange sind drei Fischerfamilien, die in Playa Oriental, Playa Pescadores und Playa del Muerto hausen.

\section{MATERIAL UND METHODEN}

Die Feldarbeiten zur vorliegenden Arbeit erstreckten sich von September 1982 bis Juli 1983. In der Bucht wurden 101 Untersuchungspunkte ausgewählt, auf die verschiedenen Substrat-Typen verteilt, und am Ufer wurden 12 Litoralprofile festgelegt, um die Zonierung festzustellen (Abb. 6). Zur Entnahme von Bodenproben in Weich- und Sandböden diente ein Van-Veen-Greifer (Kantenlänge $25 \times 20 \mathrm{~cm}$ ), mit dem in jeder Station 5 Proben entnommen wurden, so daß insgesamt eine Fläche von $0,25 \mathrm{~m}^{2}$ ausgewertet werden konnte. An einer zusätzlichen Probe wurde eine Korngrößenanalyse durchgeführt. Im Litoralbereich und in Korallenriffen wurden die Proben vom Taucher entnommen, und zwar auf Sand- und Weichböden mit einem handbedienten Kastengreifer $\left(200 \mathrm{~cm}^{2}\right)$ und von 10 nahe beieinanderliegenden Flächen. Auf Hartsubstraten (Kies-, Geröll-, Felsen-, Korallen- und Kalkalgengrund) wurde ein scharfkantiger Metallrahmen $(50 \times 50 \mathrm{~cm})$ aufgelegt bzw. eingedrückt und das Material mit einer Handschaufel in einen Stoffsack gefüllt. Für Zonierungsuntersuchungen auf Fels und Korallen diente ein PVC-Rahmen $(25 \times 100 \mathrm{~cm})$, innerhalb dessen die Mollusken abgesammelt wurden. Die Proben wurden im allgemeinen durch Siebe von $1 \mathrm{~mm}$ Maschenweite gespült und anschließend in Formalin (5\%) oder in Alkohol $(70 \%)$ bis zur späteren Auswertung im Labor konserviert.

Im Labor wurden die Mollusken von Sediment, Schalenresten und Leergehäusen gereinigt und zur Ermittlung der Biomasse naß gewogen, die kleinsten mit der Schale. Da die Sammelmethoden in den Korallenriffen quantitativ unzureichend waren, können für diesen Biotop keine Vergleichswerte gegeben werden. Korngrößenverteilung und Sortierkoeffizient wurden nach Gray (1981) ermittelt.

Nach der Bestimmung (zum Teil mit Hilfe eines Raster-Elektronenmikroskops) wurden einige der üblichen zönologischen Charakteristika errechnet: die Abundanzen, die Dominanzen, die Diversität ( $\mathrm{H}^{\prime}$, mit Logarithmus zur Basis e; nach Shannon, 1976) und die Äquität (evenness E; Haeupler, 1982). Zum quantitativen Vergleich der Molluskenbestände wurde eine für Benthos-Untersuchungen geeignete Artenidentitătsmethode benutzt (Field et al., 1982), die in letzter Zeit zunehmend verwendet wird, da sie 


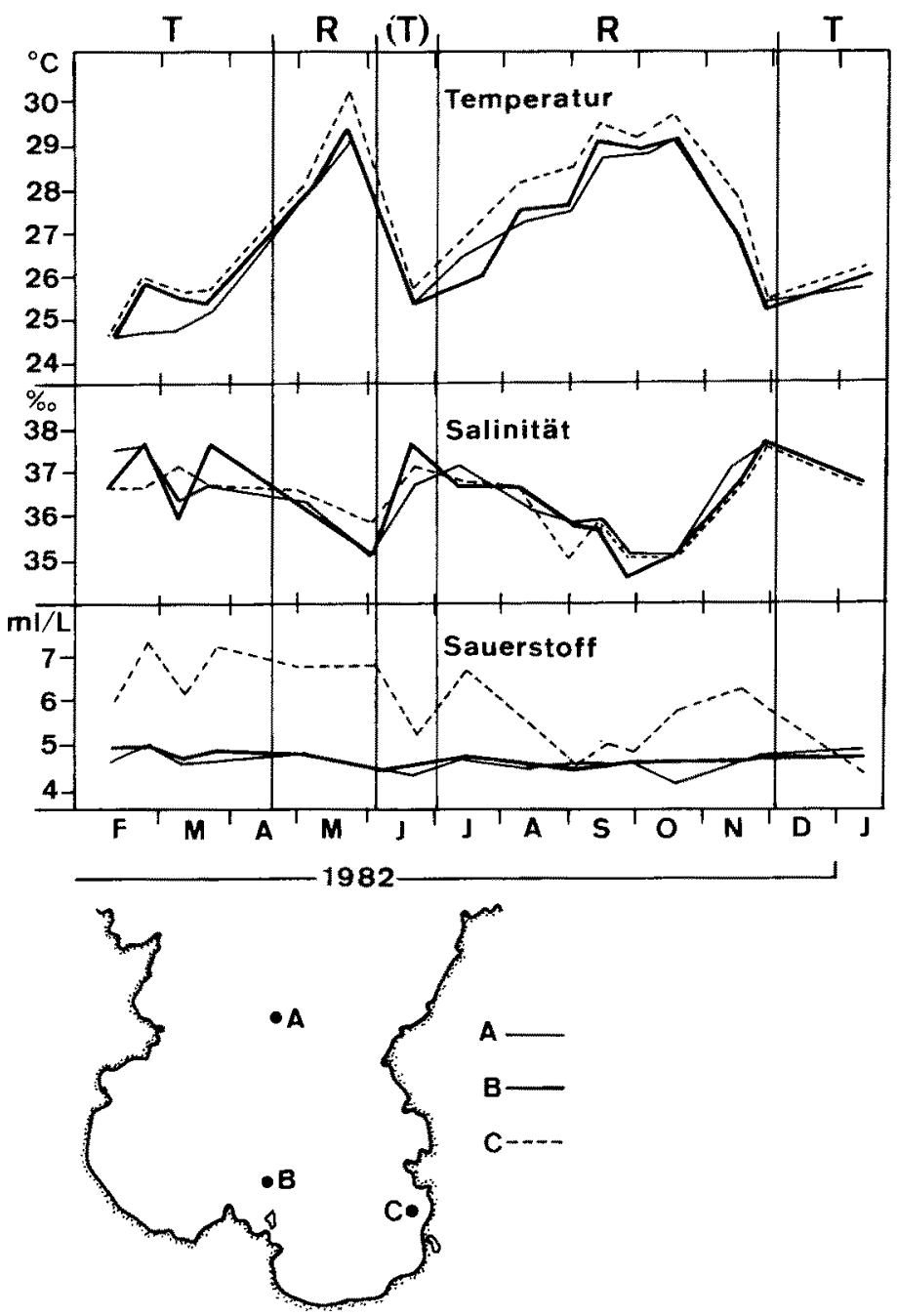

Abb. 3. Jahresverlauf von Temperatur, Salinität und Sauerstoff-Gehalt an 3 Meßstellen (A, B, C) in der Bahía de Nenguange. T: Trocken-, R: Regenzeit

ermöglicht, das Ausmaß der Ubereinstimmung im Artenspektrum von Tierbeständen zu ermitteln und anschaulich zu machen (Gray, 1981). Die ermittelte Änlichkeitsmatrix wird in ein Dendrogramm umgesetzt, das den Grad der Ahnlichkeit zwischen verschiedenen Molluskenbeständen veranschaulicht. Zur Beurteilung von Nischenbreite und Nischenüberlappung wurden die Berechnungsmethoden von Colwell \& Futuyima (1971) herangezogen. 


\section{BEFUNDE}

\section{Submarines Relief und hydrographische Faktoren}

Die Bahía de Nenguange liegt im Bereich der nordwestlichen Ausläufer der Sierra Nevada de Santa Marta, die durch orogenetische Prozesse im Pleisto-Pliozän emporgehoben wurde. Diese Ausläufer setzen sich im oberen Kontinentalschelf fort und bilden eine zerklüftete Riasküste. Die submarine Topographie ist aus Abb. $5 \mathrm{zu}$ entnehmen. Bemerkenswert ist das starke Gefälle im NO. Die steilen Bergflanken der W-Seite und östlich der Playa Brava fallen zwar fast senkrecht zum Meer ein, doch stoßen sie da bald auf eine aus Sänden bestehende Ebene, die sich als terrassenartige Plattform zur Mitte der Bucht hinzieht. Am nordöstlichen Ufer reichen die steilen Felswände dagegen bis in ca. 20-25 m Tiefe.

In den tiefen Bereichen der Bucht findet man überwiegend weichen, grauen Schlick oder schlickigen Sand mit hohem Gehalt an organischem Material, besonders unterhalb $27 \mathrm{~m}$ im mittleren Bereich der Senke. Von dort aus stellt sich aufwärts die FaziesAbfolge Schlick - Feinsand - Mittelsand - Grobsand ein. An bestimmten Stellen (an der Böschung) geschieht der Wechsel sehr schnell, so daß in enger räumlicher Nachbarschaft Sedimente auftreten, deren Korngrößen sich um zwei Zehnerpotenzen unterscheiden. Sublitoralboden und Strandwälle von Playa Brava und Playa Oriental bestehen weitgehend aus quarzreichen Feinsänden; das Sediment ist bei Playa Brava mit $0,20 \mathrm{~mm}$ Korndurchmesser etwas gröber als bei Playa Oriental $(0,16 \mathrm{~mm})$ und weist geringere Streuung auf. Mit zunehmender Tiefe werden die Sedimente vor der Playa Oriental allmählich feiner, vor der Playa Brava dagegen gröber. In der W-Hälfte der Bucht findet sich kalkreiches Substrat. Der mit geringer Neigung in 10-11 m Tiefe verlaufende Grund ist großenteils von Kalkalgen (Lithothamnion spec., Goniolithon spec.) bedeckt, Foraminifera und Rollkorallen (Siderastrea spec.) sind häufig. Dazwischen findet sich feines bis faustgroßes Geröll. Kalkreiche Sedimente in Form von Muschelschill und Korallensand sind vor allem in der Nähe von Riffen bestimmend. So bestehen die Strände von Playa Placelito, Playa del Muerto und Playa Pescadores hauptsächlich aus Korallensänden. An der Basis der Riffhänge liegt meist eine Ansammlung von Korallenbruch. Dieser Schutthalde schließt sich ein unterschiedlich breiter Grobschillgürtel an, der je nach Lage mit zunehmender Tiefe in Feinsand übergeht. Die bedeutendsten Seegrasbestände sind im Ostteil der Bucht zu finden. Die Hauptkomponente ist Thalassia testudinum, die in einem breiten Substratspektrum gedeiht: auf Schlick, Fein-, Grobsand und Kies. Seegraswiesen treten in Tiefen von wenigen cm unter der Wasseroberfläche bis zu $11 \mathrm{~m}$ auf. Die Korallen bilden entweder kleine, der Felsküste parallele Saumriffe, die höchstens 20-30 m breit sind, oder Lagunensaumriffe, die einige Dutzend Meter vor dem Ufer beginnen und sich seewärts ausbreiten. An seichten, ruhigen Stellen sowie auf dem Riffdach sind Elch- und Hirschgeweihkorallen (Acropora palmata, A. cervicornis) am häufigsten, in tieferen Lagen und am Riffhang kommen dagegen meist massive und krustenbildende Arten vor (Diploria spec., Meandrina spec., Agaricia spec.). Im allgemeinen liegt die äußere Hangbasis des Riffes in weniger als $14 \mathrm{~m}$ Tiefe; um die Roca Ahogada und am nordöstlichen Ufer zieht sich der steile Riffhang bis zu 25 m hinunter (vgl. auch Werding \& Erhardt, 1977). 


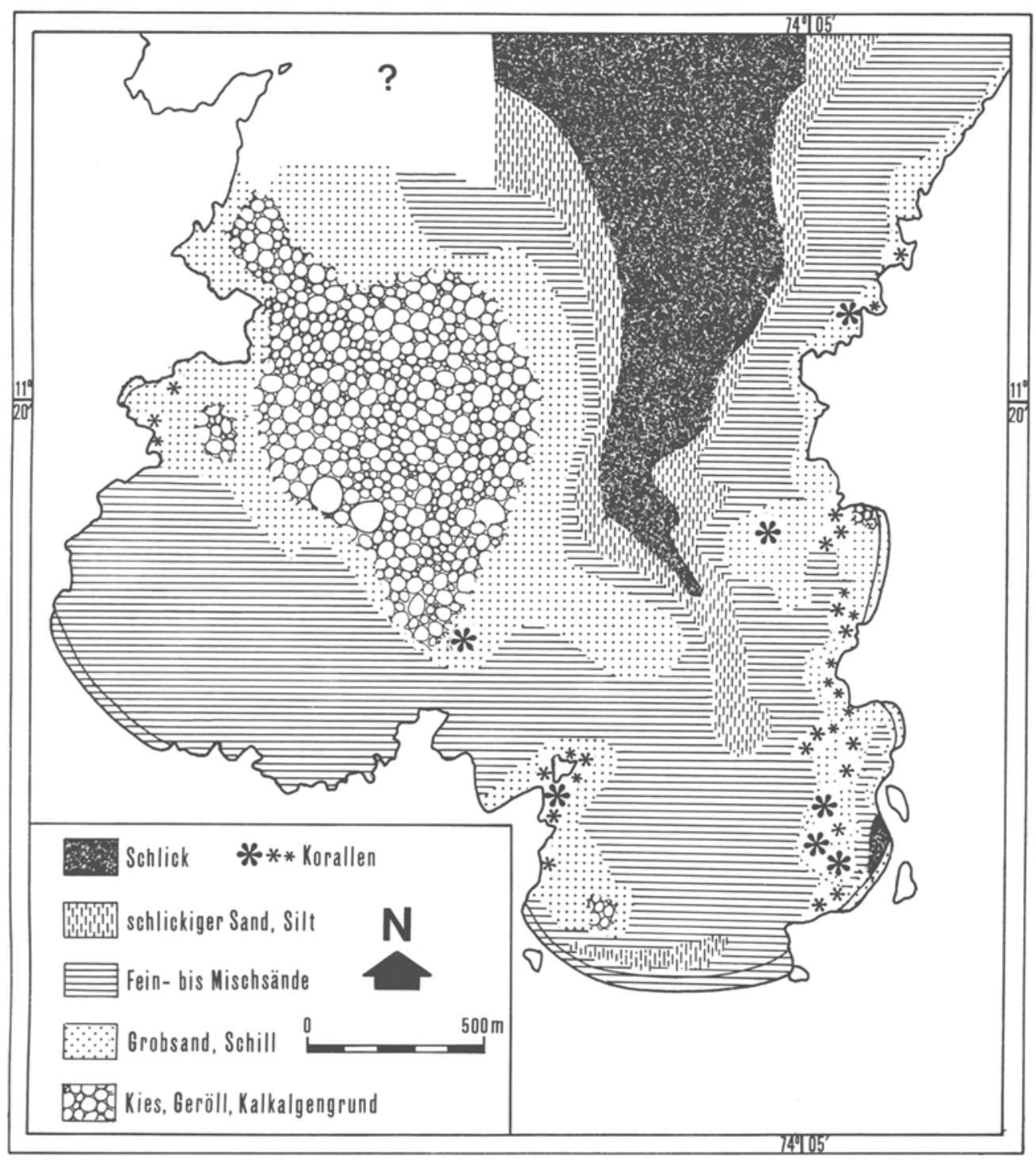

Abb. 4. Die Verteilung der Substrate in der Bucht

Aufgrund der vorherrschenden Windrichtungen bewegt sich das Oberflächenwasser in der Mitte der Bucht hauptsächlich nach SW und strömt an den O- und W-Küsten wieder nach $N$ zurück. Die ausgeprägten Schwankungen der Temperatur- und Salinitätskurven (Abb. 3) sind negativ miteinander korreliert: in der Trockenzeit dringt Auftriebswasser niedrigerer Temperatur, aber höheren Salzgehalts in die Bucht ein. 


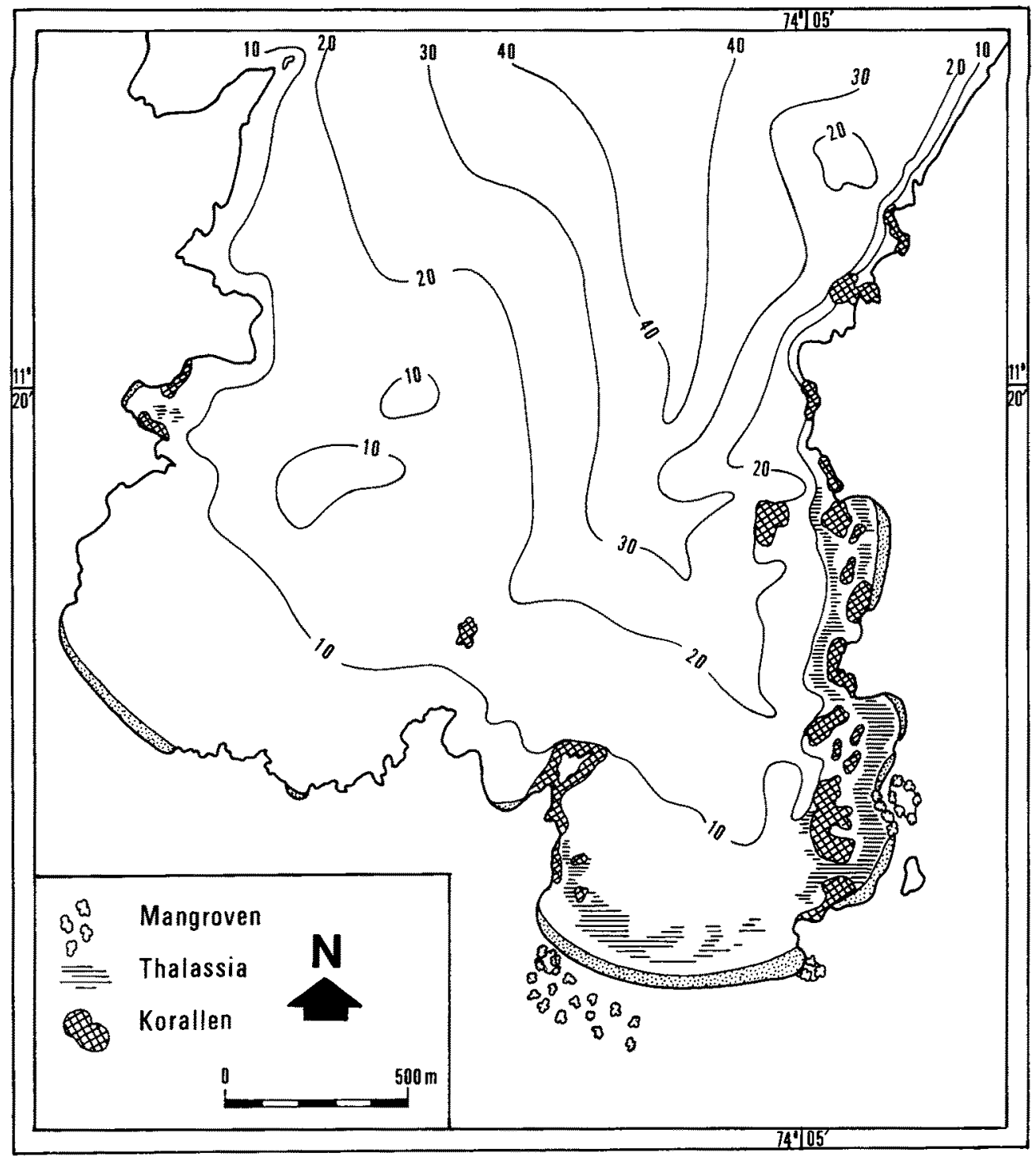

Abb. 5. Die Vertellung von Mangrove, Seegraswiesen und Korallenriffen in der Bucht sowie die Haupt-Tiefenlinien

\section{Malakozönosen}

Insgesamt wurden in der Bahía de Nenguange 489 Mollusken-Arten nachgewiesen, davon 382 lebend. Es handelt sich um 155 Bivalvia, 6 Scaphopoda, 8 Polyplacophora, 43 Archaeo-, 122 Meso-, 124 Neogastropoda und 28 Opisthobranchia (darunter 17 Pyramidellidae) sowie 3 pulmonata. Im folgenden wird die Verteilung dieser Arten auf die 


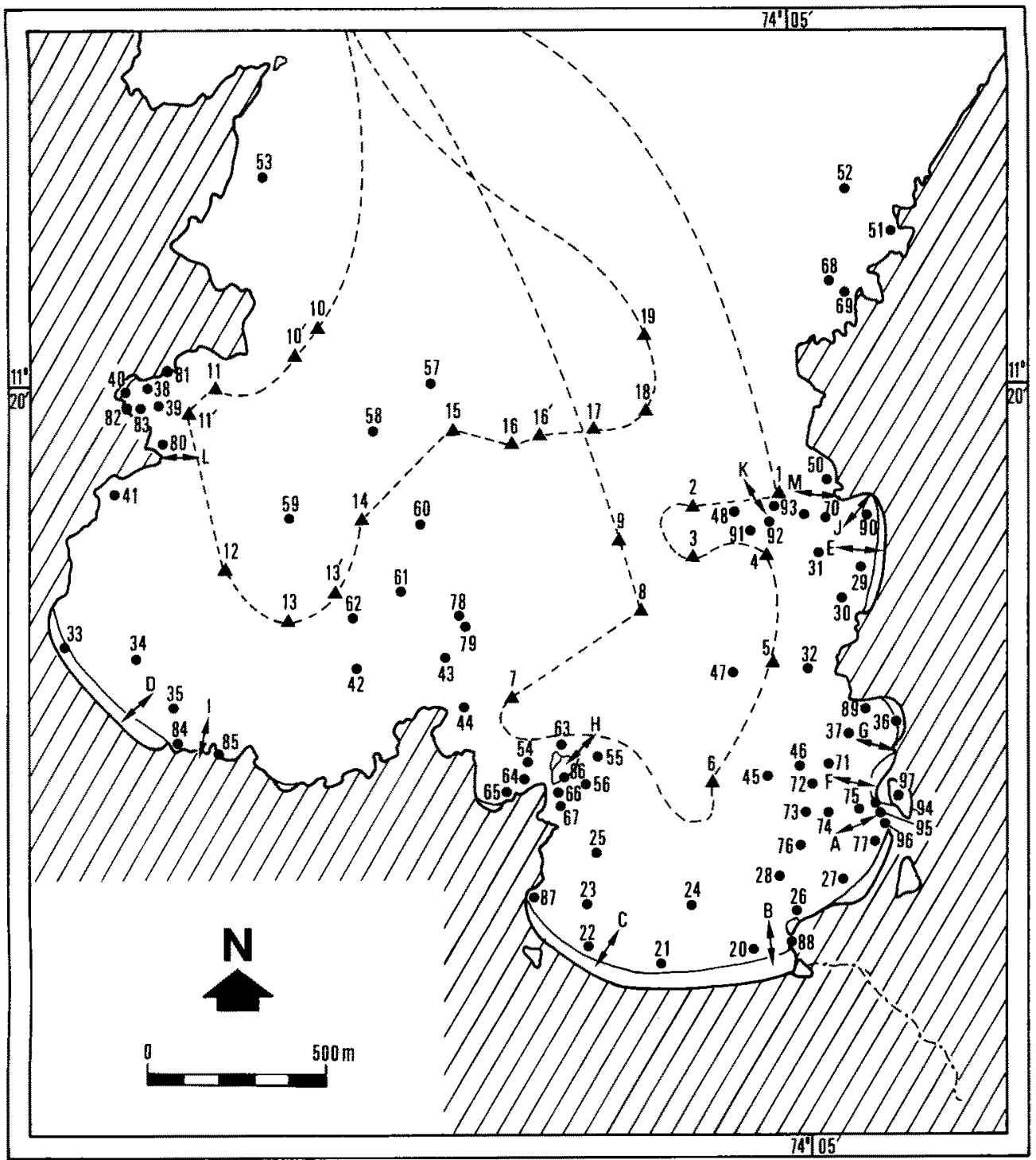

Abb. 6. Die Lage der Sammelstationen (Ziffern) und der -profile (Buchstaben) (Dreiecke bezeichnen Bodengreifer-Stationen, die gestricheiten Linien sind Tiefenmeßstrecken)

verschiedenen Substrattypen erörtert und anschließend anhand zoozönologischer Kriterien analysiert.

\section{Weichböden}

Die Mollusken der Weichböden gehören überwiegend zum Endopelos. Die beiden ersten Positionen in der Dominanzhierarchie werden durch zwei Scaphopoda eingenommen (Cadulus acus 21,4\%, Dentalium didymum 14,3\%). Bei Station 47, wo das 
Substrat etwas gröber ist, dominierte Tellina consobrina (19\%), gefolgt von Cadulus acus $(13,7 \%)$, Dentalium disparile $(12,5 \%)$ und $D$. didymum $(8,5 \%)$. In allen Proben waren außerdem anzutreffen Olivella perplexa, die in den oberen Sedimentschichten lebt, sowie Nucula dalmasi und Limopsis antillensis. Im selben Habitat kamen regelmäBig Polychaeta vor. Die Biomassewerte für die Mollusken lagen zwischen 3,35 und $5,11 \mathrm{~g} / \mathrm{m}^{2}$. Nur an Station 47 mit zahlreichen Anadara notabilis wurden $9,8 \mathrm{~g} / \mathrm{m}^{2}$ erreicht. Von den insgesamt 37 erfaßten Molluskenarten in diesem Weichboden waren 5 in allen Proben enthalten(Cadulus acus, Dentalium didymum, Nucula dalmasi, Olivella perplexa, Turbonilla pusilla), während 14 Arten in nur je 1 Probe vertreten waren. Hinsichtlich der Artenzusammensetzung stehen sich die Stationen 18 und 19 mit 14 gemeinsamen Arten sowie 3 und 47 mit 11 gemeinsamen Arten am nächsten. Da sich die untersuchten Weichböden im tiefsten Teil der Bucht befinden, enthielten die Proben auch zahlreiche Leergehäuse von Arten, die in diesen Bereich eingeschwemmt worden sind.

\section{Fein- und Mischsande}

Fein- und Mischsandböden sind in der Bahía de Nenguange besonders weit verbreitet. Grob- und Korallensand, oft mit Schill gemischt, finden sich vor allem in der Umgebung von Riffbauten und Kalkalgen-Hartgrund. Die Artenzusammensetzung ist auf den genannten Substraten so verschieden, daß sie getrennt besprochen werden müssen. Fein- und Mischsande reichen, von den Strandwällen der Playa Oriental und der Playa Brava ausgehend, bis in etwa $20 \mathrm{~m}$ Tiefe in die Bucht hinein, wo sie dann allmählich in Schlicksand übergehen. Die Eulitoralzone der Playa Brava ist durch die geringe Strandböschung und die relativ starke Brandung gekennzeichnet; sie ist besonders breit. Nach dem Durchfeuchtungsgrad lassen sich folgende Abschnitte in ihr unterscheiden: die Brandungszone, die ständig vom Wellenschlag aufgewühlt wird, und die Waschzone mit einem gesättigten Bereich, der stets von einem Wasserteppich bedeckt ist, sowie einem ungesättigten Bereich, in dem das Wasser der Wellen im Sand versickert. In der darüberliegenden Spritzwasserzone wurden zwar Amphipoda und Isopoda, aber keine Mollusken angetroffen. In der ungesättigten Waschzone lebt nur Donax denticulatus (Abb. 7), vor allem in der W-Hälfte der Playa Oriental. Im gesättigten Bereich dominiert die genannte Art. Dazu kommt in der Playa Brava Terebra cinerea, in kleineren Bereichen lokalisiert (kumulare Dispersion). Die Dominanzverhältnisse beider Arten verschieben sich daher je nach Standort. Die Brandungszone ist durch den abrupten Abundanz-Rückgang von $D$. denticulatus charakterisiert. Ein erneuter Abundanz-Anstieg dieser Muschel im unteren Bereich der Brandungszone ist offenbar auf von der Dünung versetzte Individuen zurückzuführen, da diese sich meist auf der Sandoberfläche befanden und nicht eingegraben waren.

In der Brandungszone der Playa Oriental fehlt $T$. cinerea im allgemeinen; sie ist nur an der O-Seite dieses Strandabschnittes in 5-30 cm Wassertiefe häufig (bis zu 25 Tiere pro $\mathrm{m}^{2}$ ). Das Siedlungsgebiet beschränkt sich auf die letzten $50-60 \mathrm{~m}$ der O-Hälfte des Strandes. Dort kommt es bei stärkeren Regenfällen zu einem Durchbruch des Strandwalles, so daß Süßwasser aus der dahintergelegenen Lagune ins Meer gelangt. In der Regenzeit wird die Population stark reduziert (auf ca. 0,5 bis 1,5 Individuen $/ \mathrm{m}^{2}$ ); im Januar erreicht sie wieder den ${ }_{2}$ normalen" Stand (10-15 Tiere/ $\mathrm{m}^{2}$ ), besteht dann aber nur aus $17-25 \mathrm{~mm}$ großen Jungtieren. Diese Population von $T$. cinerea weist also die 


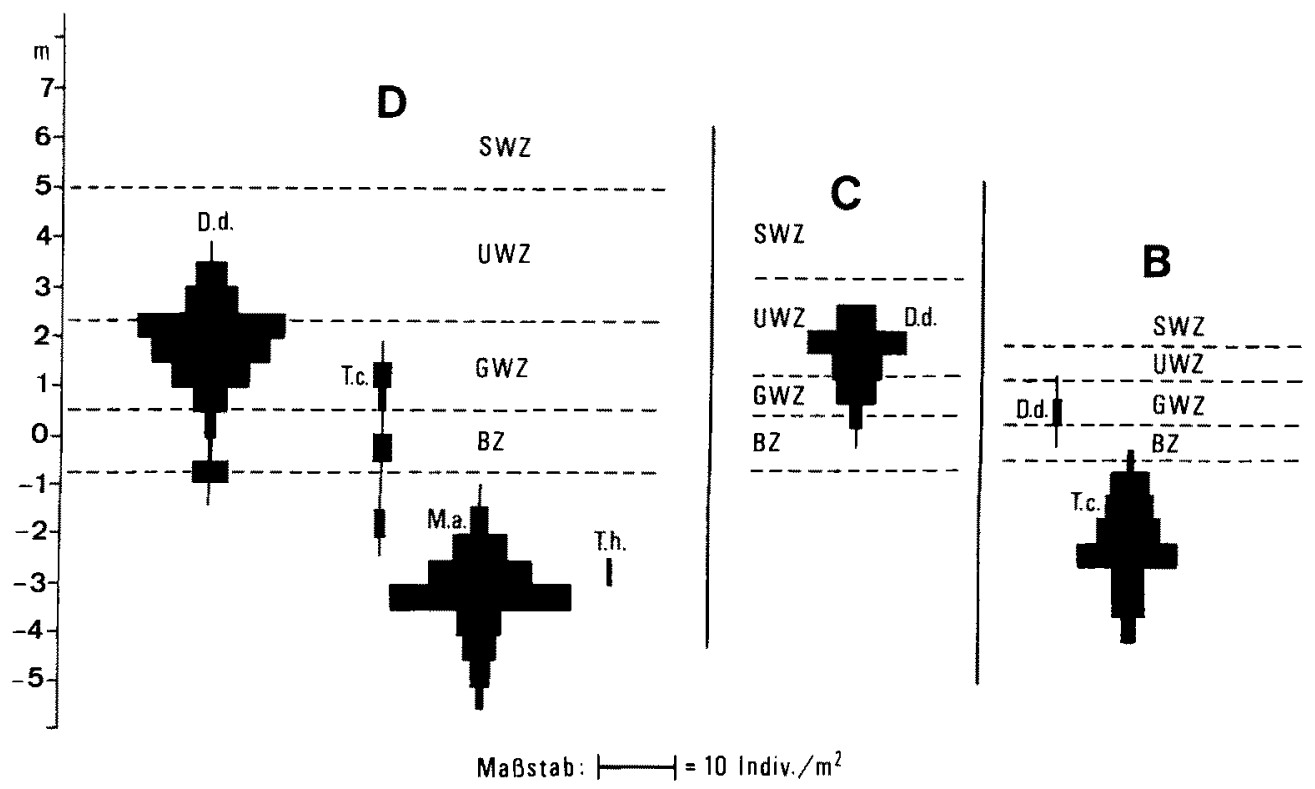

Abb. 7. Abundanzen einiger Molluskenarten in Profilen an der Playa Brava (D) und an der Playa Oriental (C: Westen, B: Osten). BZ: Brandungszone, GWZ: gesättigte, UWZ: ungesättigte Waschzone, SWZ: Spritzwasserzone. D.d.: Donax denticulatus, M.a.: Mazatlania aciculata, T.c.: Terebra cinerea, T.h.: Terebra hastata

Merkmale einer Zyklozönose auf, deren Struktur durch die Periodizität von Regen- und Trockenzeiten bestimmt wird.

Unterhalb der Brandungszone der Playa Brava verschwindet $T$. cinerea allmählich; dafür wird Mazatlania aciculata die dominierende und oft sogar die einzige Art (15-25 Tiere $/ \mathrm{m}^{2}$ ). In den muldenartigen Vertiefungen des Bodens liegt die Abundanz besonders hoch $\left(30-45 / \mathrm{m}^{2}\right)$. Weiter in die Tiefe nimmt die Häufigkeit allmählich ab, ohne daß dafür andere Arten auftreten. Erst unter 1,5 m Wassertiefe siedelt dann eine aus der Tiefe hochreichende, hier verarmte Gemeinschaft von Gouldia cerina $\left(4-8 / \mathrm{m}^{2}\right)$, Ervilia nitens $\left(4-6 / \mathrm{m}^{2}\right)$ und Olivella adelae $\left(2-6 / \mathrm{m}^{2}\right)$.

Im westlichen Teil der Playa Oriental, wo das Sediment relativ hohe Anteile an Schlamm aufweist und Thalassia spärlich gedeiht, lebt eine größere Anzahl von Molluskenarten als am Ostende (Profil B, Station 20), wo neben $T$, cinerea 17 Molluskenarten gefunden wurden. Mit hoher Abundanz treten Olivella minuta, Mitrella lunata, Terebra quadrispiralis, Acteocina candei, Polinices lacteus und Nassarius albus auf, während Olivella perplexa, Bulla striata, Codakia pectinella und Lucina multilineata nur im Schlammsand vorkommen. In unmittelbarer Nachbarschaft einer Seegraswiese wurden auch zwei typische Thalassia-Bewohner gefunden: Acmaea pustulata f. pulcherrima und Smaragdia viridis (Station 22). Aufgrund der kleinräumlich verschiedenen Lebensbedingungen an der Playa Oriental und der entsprechend wechselnden Molluskenbesiedlung kann das obere Sublitoral hier als Lebensraum einer Mischgemeinschaft angesehen werden, die sich an die oben besprochene Zonierung im Bereich der Brandungszone nach unten anschließt. Mit zunehmender Tiefe wird diese Zönose 
immer einheitlicher. Dabei verschwinden einige Arten wie z. B. Olivella minuta, andere werden häufiger (vgl. Abb. 8).

In Fein- und Mischsand, aber auch in anderen Sand- und in Weichböden sind die Gastropoden Diastoma varium und Alaba incerta stark vertreten. Ähnliches gilt für Finella dubia, Mitrella lunata, Acteocina candei und Ervilia nitens. Als Charakterarten kommen jene in Betracht, die meist nicht eudominant auftreten, aber hohe Konstanz bzw. Präsenz aufweisen. Das sind in Fein-" und Mischsand vor allem Persicula interruptolineata, Olivella adelae und Diplodonta punctata, ferner Nuculana acuta und Nucula venezuelana. $P$. interruptolineata und $O$, adelae kriechen wenige Millimeter unter der Sedimentoberfläche umher und treten mit sehr unterschiedlichen Abundanzen auf (Persicula 4-40, Olivella 14-40 Individuen $/ \mathrm{m}^{2}$ ). N. acuta, Diplodonta punctata und Acteocina candei zeigen eine leichte Präferenz für feinsandigen Boden mit Schlammanteilen (Stationen 6, 24, 45), während Nucula venezuelana, Crassinella guadelupensis, C. cf. martinicensis und Semelina nuculoides gröbere Sedimente vorziehen.

Die Biomasse-Werte zeigen kleinräumliche Unterschiede: am höchsten sind sie in unmittelbarer Nähe eines Thalassia-Bestandes (Station $24 ; 6,15 \mathrm{~g} / \mathrm{m}^{2}$ ), am niedrigsten an Station $42\left(0,78 \mathrm{~g} / \mathrm{m}^{2}\right)$.

\section{Grobsand}

Grobsand kommt in der Bahía de Nenguange vorwiegend als Korallensand vor, der je nach Lage mit Kies, Kalkalgen und Muschelschill vermischt sein kann. Reiner, weißer Korallensand findet sich im Supra- und Eulitoral von Playa Placelito, Playa del Muerto und Playa Pescadores. Außer einigen Crustacea (Ocypode spec., Pagurus spec, Emerita spec., einigen Amphipoda und Isopoda) wurden hier keine Tiere gefunden. Nur in der schmalen Waschzone am Südende der Playa Viva leben vereinzelt Heterodonax bimaculatus $\left(5-8 / \mathrm{m}^{2}\right)$. Im oberen Sublitoral bis etwa $10 \mathrm{~m}$ Tiefe ist der Grobsand meist mit Thalassia bewachsen oder von Korallenbruch und Geröll bedeckt. Einige isolierte Korallensandflecken oberhalb $3 \mathrm{~m}$ Tiefe vor der Playa del Muerto sind artenarm. Es dominieren hier die mesopsammalen Mikrogastropoden Caecum regulare (42-71 Tiere/ $\left.\mathrm{m}^{2}\right)$ und $C$. floridanum $\left(18-36 / \mathrm{m}^{2}\right)$; stellenweise finden sich Gouldia cerina, Diplodonta semiaspera und Olivella spec.

Im November und Dezember 1982 wurden in 2-4 m Tiefe vor der Playa Placelito einige adulte Cassis tuberosa beobachtet, und zwar etwa 14 Exemplare auf einer Fläche von ca. $30 \mathrm{~m}^{2}$. Im Januar 1983 konnte kein Tier dieser Art hier nachgewiesen werden, ein Individuum fand sich erst in $10 \mathrm{~m}$ Tiefe. Das spricht dafür, daß die Population größere Vertikalwanderungen durchführt.

Mit zunehmender Tiefe verringert sich der Korallensandanteil im Sediment, und die durchschnittliche Korngröße steigt an. Dazu kommt ein wachsender Anteil von Schill, Kalkalgen und Korallenbruch sowie größeren Schwammarten (u. a. Cinachyra spec., Ircinia spec.) und Rollkorallen (Porites spec., Siderastrea spec.). In diesem Mischbiotop lebt eine große Vielfalt an Molluskenarten, viele allerdings mit geringer Wohndichte.

Abb. 8. Durchschnittliche Dominanzwerte für die häufigsten Molluskenarten auf bestimmten Substraten. 1. und 2. Reihe: Fein- und Mischsandböden; 1. Reihe links: Station 24, rechts: Station 6; 2. Reihe links: Station 7, rechts: Station 13; 3. Reihe: Grobsand-Schill: links Durchschnitt der Stationen 1, 4, 5, 13',16' und 17, rechts Station 8; unten: Schlickboden der Senke (Durchschnitt) 

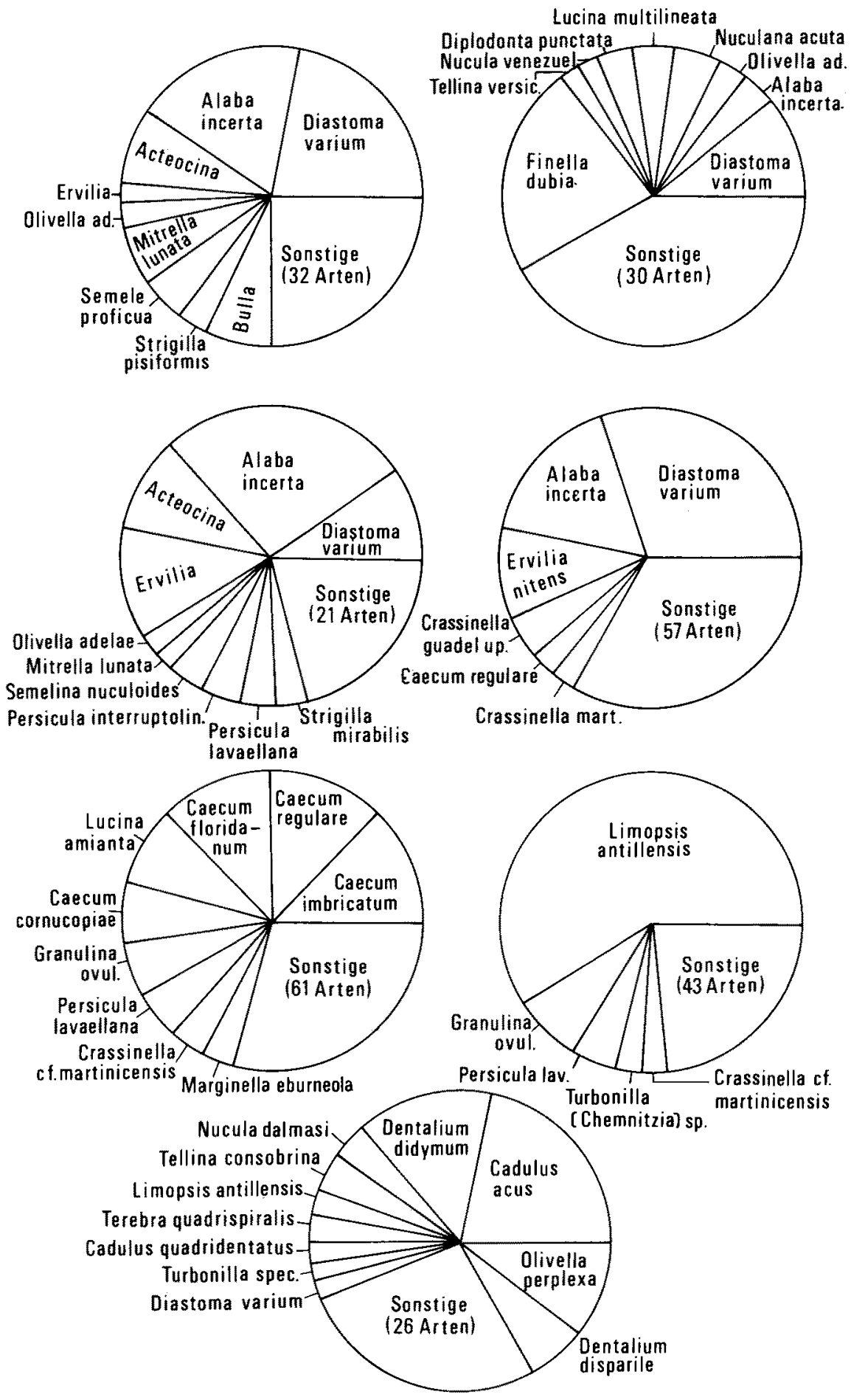
Die häufigsten Arten unter den Makromollusken sind Trachycardium isocardia, T. muricatum, Tellina listeri, Glycymeris pectinata und Divaricella quadrisulcata. Außerdem wurden frische Leerschalen von Tellina fausta, Papyridea soleniformis, Chione cancellata mazycky und Semele purpurascens gefunden. Unter den mesopsammalen Mikrogastropoden waren Caecum regulare, $C$. floridanum und $C$. imbricatum am häufigsten (100-170 bzw. $50-70 \mathrm{bzw}$. 12-60 Tiere/ $\left.\mathrm{m}^{2}\right)$. In überwiegend korallensandigem, weitgehend schillfreiem Boden traten Corbula caribaea, Persicula lavaellana, Crassinella martinicensis und Tellina sybaritica auf. Dagegen ziehen Triphora turristhomae, T. pulchella und T. nigrocincta sowie Marginella eburneola gröbere Sedimente vor. Die Biomasse-Bestimmungen ergaben sehr unterschiedliche Werte. Am niedrigsten lagen diese an Station $32\left(3,9 \mathrm{~g} / \mathrm{m}^{2}\right)$, wo ausschließlich Kleinarten gefunden wurden. Die höchsten Werte wurden an Station 31 ermittelt $\left(10,3 \mathrm{~g} / \mathrm{m}^{2}\right.$; mit Trachycardium isocardia und Periploma spec.) sowie an Station $32\left(8,7 \mathrm{~g} / \mathrm{m}^{2}\right.$; mit Glycymeris pectinata und Divaricella quadrisulcata).

Tiefer gelegene Areale mit reinem Grob- bzw. Korallensand treten in der Nenguange kaum auf. Unterhalb $10 \mathrm{~m}$ Tiefe sind grobe Sedimente grundsätzlich nur in Form von Muschelschill zu finden, der mit Mineralsänden, Kalkalgen, Bryozoa- und Foraminifera-Skeletten in unterschiedlichem Ausmaß vermischt ist. Die Molluskenvielfalt in diesem Substrat ist außerordentlich groß (mindestens 16 Arten auf $0,05 \mathrm{~m}^{2}$ ). Mit Ausnahme der Station 8 wurden an allen anderen Stationen Kleinschnecken der Gattung Caecum als dominierend gefunden, darunter C. imbricatum mit 1050 Individuen $/ \mathrm{m}^{2}$. Unter den Muscheln ist Carditopsis smithii sehr häufig. Ziemlich regelmäßig waren Granulina ovuliformis, Alvania auberiana, Persicula lavaellana, Turbonilla pusilla sowie Crassinella cf. martinicensis, C. Iunulata, Nucula venezuelana, Corbula caribaea, Gouldia cerina und Tellina sybaritica anzutreffen. Von den größeren Arten wurde die Schnecke Cantharus lautus in etwa $30 \%$ der Proben mit je 1-2 Individuen je Probe nachgewiesen. Limopsis antillensis, die lebend sonst fast ausschließlich im Schlick gefunden wurde, erreichte in den vier in Station 8 (24 m Tiefe) entnommenen Proben sehr hohe Abundanzen (im Mittel 2600 Tiere $/ \mathrm{m}^{2}$ ). Bemerkenswert ist, daß im selben Habitat keine der Caecum-Arten eudominant auftrat und daß Carditopsis smithii völlig fehlte. Uberdurchschnittlich häufig waren dagegen Crassinella Iunulata und Granulina ovuliformis. Mit Ausnahme der Station 8 stimmten Artenzusammensetzung und Dominanzverhältnisse an den Schill-Grobsand-Stationen überein (Abb. 7). Die Biomasse wies geringe Differenzen auf, sowohl zwischen den Proben an einer Station wie auch beim Vergleich mehrerer Stationen miteinander: $12,1 \mathrm{~g} / \mathrm{m}^{2}$ an Station $8,6,78 \mathrm{~g} / \mathrm{m}^{2}$ an den übrigen Untersuchungspunkten.

\section{Seegraswiesen}

Seegraswiesen sind weit verbreitet. Während Thalassia testudinum auf Kies-, Schill- und Grobsandböden allein auftritt, vermischt sich diese Art auf feineren Sänden örtlich mit Syringodium filiforme. Der größte Seegrasbestand nimmt den Sektor der Rifflagune vor der Playa Pescadores und der Playa Viva ein, kleinere Bestandsflächen finden sich an mehreren Stellen. Neben einigen ubiquitären Arten (z. B. Alaba incerta, Diastoma varium, Persicula lavaellana) wurde regelmäßig die epiphytisch lebende Smaragdia viridis viridemaris angetroffen, die von den Thalassia-Blättern die aufsitzenden Algen abweidet. Ebenfalls epiphytisch und stellenweise sehr häufig ist eine kleine, 
bernsteinfarbene Form von Acmaea leucopleura. Die Lucinacea dominierten an allen untersuchten Seegrasstandorten; sie sind infaunische Bivalvia, die mit ihren langen Ingestionssiphonen suspendierten Detritus aufpipettieren. Von den 18 in ThalassiaBeständen erfaßten Muschelarten gehören 12 zu den Lucinacea (9 Lucinidae, 3 Ungulidae). Sie machen $20 \%$ der Gesamtindividuenzahl der Mollusca aus. Stellenweise erreicht die Dominanz der Lucinacea $80 \%$, im Durchschnitt beträgt sie etwa $60 \%$. Besonders häufig sind Codakia costata, $C$. orbicularis, $C$. orbiculata, $C$. pectinella und Lucina multilineata. In 8 der 13 untersuchten Thalassia-Standorte wurden mindestens 3 dieser 5 Lucinidae zusammen angetroffen. Die 3 erstgenannten Arten waren in allen oberhalb $1 \mathrm{~m}$ Wassertiefe gelegenen Probestellen vertreten. Dagegen sind die Ungulidae Diplodonta punctata und $D$. semiaspera häufiger unterhalb $2 \mathrm{~m}$ anzutreffen. Andere, regelmäßig vorkommende Muscheln sind Chione cancellata, Gouldia cerina, Semele proficua sowie an einigen Fundorten Mactra fragilis und Tellina versicolor.

In und auf den obersten Sedimentschichten lebt eine große Anzahl von Gastropoda. Von Diastoma varium wurden zwischen 45 und 460 Individuen $/ \mathrm{m}^{2}$ nachgewiesen. Andere Arten, die offenbar in den detritusreichen Seegraswiesen ihr optimales Habitat finden, sind die Mikrogastropoden Alaba incerta, Persicula lavaellana, Acteocina candei, Rissoina bryerea und Odostomia laevigata. Unter den größeren Arten waren Bulla striata, Cerithium eburneum, Modulus modulus, Columbella mercatoria, Engoniophos unicinctus und Nassarius vibex in mehr als $25 \%$ der Proben vertreten. Die Wohndichte von Strombus raninus wurde an verschiedenen Stellen zwischen 0,4 und 1,5 m Wassertiefe auf 3,2 bis $0,5 \mathrm{Tiere} / \mathrm{m}^{2}$ geschätzt. Juvenile Strombus gigas kommen in unregelmäBiger Verteilung vor. In den Proben nicht quantitativ erfaßt wurden Strombus pugilis, Turritella variegata und Cypraecassis testiculus. Auffällige epifaunische Bivalvia sind Arcopsis adamsi und juvenile Chamidae, besonders häufig auf Modiolus americanus und Anadara notabilis, allerdings nur als Leerschalen. In wenigen Proben wurde die sich einige Millimeter eingrabende Solemya occidentalis gefunden. Im allgemeinen weisen die höher gelegenen Teile der Thalassia-Wiesen mehr charakteristische Faunenelemente auf als die tieferen. Das gilt nicht nur für die Mollusken, sondern auch für andere Tiergruppen. So wurden der Seeigel Lytechinus variegatus, einige Holothuria-Spezies sowie Spinnenkrebse (u. a. Mithrax coryphe und sculptus) stets oberhalb $1,5 \mathrm{~m}$ Wassertiefe beobachtet. Eine stark vereinfachte Thalassia-Gemeinschaft des seichten Wassers ist in Abb. 9 dargestellt.

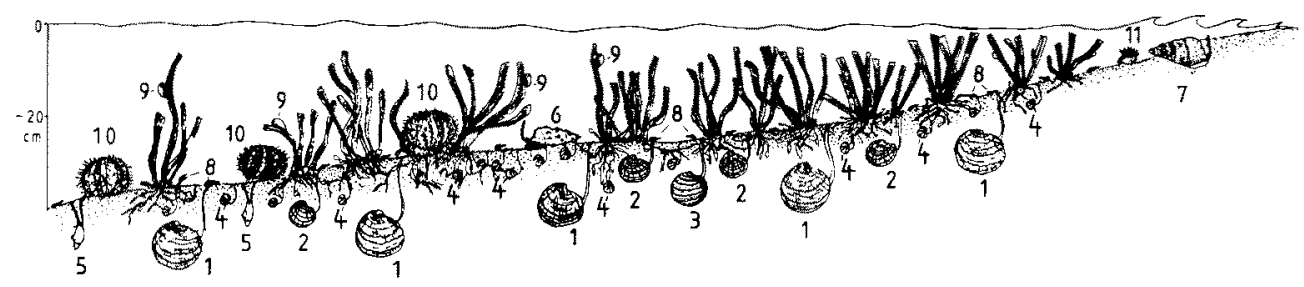

Abb. 9. Vereinfachte Darstellung der Lebensgemeinschaft der seichten Thalassia-Wiese. 1: Codakia orbicularis, 2 : C. orbiculata, 3: Lucina pennsylvanica, 4: kleine Muscheln (wie Codakia costata, Lucina muricata, juvenile Tellina fausta u.a.), 5. Tellina versicolor, 6.: Cerithium eburneum, 7 ; Nassarius vibex, 8: Mikrogastropoden der Epifauna (wie Alaba incerta, Diastoma varium, Acteocina candei, Rissoina bryerea, Acteon punctostriatus u.a.), 9: Smaragdia viridis viridemaris, 10: Lytechinus variegatus (Echinoidea), 11: Aktinien 


\section{Primäre Hartsubstrate}

Das primäre Hartsubstrat besteht im wesentlichen aus Schiefer (an der Felsküste = $75 \%$ des Ufers), aus einigen felsigen Untiefen und erratischen Blöcken vor Playa Placelito und östlich der Playa Brava. Bis in etwa $5 \mathrm{~m}$ Wassertiefe ist es an einigen Stellen nicht besiedelt, während es in tieferen Schichten von einer Fülle sessiler Organismen so dicht bewachsen ist, daß diese selbst als Substrat für andere fungieren. Die Zonierung im Felslitoral hängt im wesentlichen von der Wellenexposition ab: hier werden 5 Kategorien unterschieden. Die am stärksten exponierte Kategorie 5 war wegen der Steilheit des Ufers und der Stärke der Brandung nicht zugänglich; Standorte der Kategorie 4 waren nur an besonders ruhigen Tagen zu untersuchen. Als Referenzlinie wurde die von Brattström (1980) festgelegte Bezugslinie (zero level) benutzt. Die Zonen am Felsufer sind wegen ihrer unterschiedlichen Färbung mit bloßem Auge erkennbar. Um die Ergebnisse leichter mit denen von Brattström bei Santa Marta vergleichen zu können, wurde hier dieselbe Terminologie verwandt.

Das obere Sublitoral ist meist von Korallen und anderen sessilen Organismen bewohnt. "Nackter" Fels ist von einer Lithothamnion-Schicht überzogen; an einigen Stellen sind dort Sargassum spec. und andere Algen angeheftet. Außer dem Seeigel Echinometra lucunter, einigen Schwämmen, Ascidien und Hydrozoen wurden an diesen Stellen keine weiteren tierischen Bewohner festgestellt. An anderen Standorten (z. B. M) sind Leucozonia nassa, Thais deltoidea und rustica bis $2 \mathrm{~m}$ Tiefe sehr häufig. An der Südseite der Roca Ahogada treten oft Conus mus und Morula nodulosa auf. Die einzigen Bivalvia sind hier Chama sinuosa, macerophylla und Pseudochama radians. Die Schalen der genannten Chama-Arten weisen in etwa $60 \%$ der Fälle Bohrlöcher von Lithophaga aristata auf.

Die Grenze zwischen Sub- und Eulitoral ist anhand der Besiedlung im allgemeinen nicht zu erkennen. Es ist ein Überlappungsgebiet ausgebildet; nur am Westufer der Nenguange kann die obere Verbreitungsgrenze von Sargassum als Indikator verwendet werden, ähnlich wie bei Santa Marta (Brattström). Im übrigen sind andere Organismen zur Definition der Grenze heranzuziehen, wie großflächige Kolonien von Zoanthus und Patythoa oder das Vorkommen von Echinometra lucunter.

Im Eulitoral entspricht die Molluskenbesiedlung der von Brattström angegebenen Zonierung. In den unteren, an das Sublitoral anschließenden Bereichen der Mischalgenzone sind neben hemisessilen Arten wie Fissurella nodosa, Hemitoma octoradiata, Diodora listeri und Acmaea leucopleura auch Mitrella ocellata und Nitidella laevigata zu finden. Etwas höher treten im Mischalgenbereich neben den genannten Arten Cittarium pica, Purpura patula, Leucozonia ocellata, Cantharus tinctus, Acmaea pustulata und antillarum sowie Chiton marmoratus auf. Thais haemastoma floridana wurde nur an Felsen am Ostende der Playa Oriental angetroffen. An Stellen, an denen der Algenteppich dünner wird oder nur noch einzelne Algenbüschel am Felsen ansitzen, kommen Acanthopleura granulata und Littorina meleagris, vereinzelt auch Petaloconchus varians und Nodilittorina interrupta vor. Felstümpel in diesem Bereich beherbergen in der Regel eine dem oberen Sublitoral oder der unteren Mischalgenzone entsprechende Zönose mit Leucozonia ocellata, Cantharus tinctus, Mitrella ocellata und Thais rustica, die in großer Anzahl auftreten kỏnnen.

An mäßig bis stark exponierten Felsufern findet man eine schmale (bis $20 \mathrm{~cm}$ 
breite), dunkle Zone, die nur von wenigen Organismen besiedelt und nur kurzfristig vom Wasser überspült wird. Neben Balaniden (Tetrachita spec., Chthamalus spec.) leben hier vereinzelt Acanthopleura granulata, Chiton marmoratus, Nodilittorina interrupta und Petaloconchus varians, selten auch Nodilittorina ziczac, in feucht bleibenden Spalten Isognomon bicolor und Brachidontes exustus.

Auch der nicht bewachsene, "weiße" Bereich (naked zone) ist in der Regel schmal und nicht überall ausgebildet. Auf wenig geneigten oder horizontal verlaufenden Felsabschnitten ist oberhalb des Balaniden-Vermetiden-Bereichs eine durch ihren Cyanophyceen-Uberzug hell erscheinende Fläche zu erkennen, die feucht, stellenweise auch naB ist. Hier sind Chiton marmoratus und Nodilittorina interrupta noch ziemlich häufig, an den nassen Stellen Nerita tessellata und versicolor sowie Petaloconchus varians, an trockenen findet sich Acanthopleura granulata. Nodilittorina ziczac erreicht Wohndichten von über 30 Tieren $/ \mathrm{m}^{2} ; N$. interrupta ist meist in kleinen Gruppen anzutreffen, die sich an nassen, schattigen Stellen versammeln. An den höher gelegenen, trockenen Stellen dieses Bereichs nimmt die Anzahl von N. angustior schnell zu, die Spalten und Löcher besiedelt.

In der Spritzwasserzone nimmt die Artenzahl rapide ab. In ihrem unteren Bereich sind Petaloconchus varians und Isognomon bicolor in Ritzen noch häufig anzutreffen, die Schnecken werden nach oben hin seltener. Nodilittorina angustior ist in der oberen Hälfte oft die einzige Molluskenart; gelegentlich wurde $N$. tuberculata festgestellt. In temporären Spritzwasserpfützen halten sich Isognomon bicolor und Petaloconchus varians am besten; Mitbewohner, wie Littorina meleagris und mespillum und die hier seltene Nerita tessellata, ziehen sich beim Austrocknen aus diesem Habitat zurück.

Im Supralitoral oberhalb der Spritzwasserzone ist vereinzelt noch $N$. angustior zu finden. Der für diesen Lebensraum sonst typische Tectarius muricatus ist in der Nenguange wie im ganzen Gebiet um Santa Marta ziemlich selten und wurde in der Bucht nur an einem Standort (G) angetroffen.

Ein aus Kies und Geröll bestehender Strandabschnitt am Nordende der Playa del Muerto $(\mathrm{J})$ beherbergt im oberen Sublitoral eine artenreiche Gemeinschaft mit z. T. charakteristischen Arten. Besonders häufig sind hier Tegula fasciata und lividomaculata, Rissoina bryerea, Cerithium litteratum und eburneum, Engina turbinella, Columbella mercatoria, Isognomon radiatus und die Käferschnecken Ischnochiton purpurascens, striolatus und pseudovirgatus. Im Flacheren nimmt die Artenvielfalt rasch ab. Typische Bewohner des Eulitoral sind Planaxis lineatus und nucleus, Mitrella ocellata, Nitidella laevigata, Puperita tristis und Nerita tessellata. Die einzige Art im oberen Eulitoral ist Nerita fulgurans, die sich an noch feuchten Stellen unter Steinen aufhält.

\section{Sekundäre Hartsubstrate}

Sekundäres Hartsubstrat wird im wesentlichen durch Kalkalgen, Korallen, Mangrove und gestrandete Baumstämme repräsentiert. Kalkalgengrund ist weit verbreitet und setzt sich u. a. aus Corallina, Halimeda und Bryothamnion zusammen, ergänzt durch Rollkorallen. Häufigste und oft einzige Muschelart ist hier Crassinella martinicensis, vereinzelt kommen Gouldia cerina und Ervilia nitens vor. Ferner wurden frische Leerschalen von Trachycardium isocardia, Glycymeris decussata und Ventricolaria rigida festgestellt, besonders auf schillreichem Grund, während in Kalkalgen eingebohrt 
Botula fusca und Lithophaga aristata, auf der Unterseite größerer Kalkalgen angeheftet Arca zebra und imbricata, Barbatia candida und domingensis gefunden wurden. Ischnochiton papillosus ist hier oft eudominant und stellenweise mit mehr als 60 Individuen $/ \mathrm{m}^{2}$ vertreten. Von vagilen Gastropoden sind besonders Tricolia bella, Turbo castanea und Triphora turristhomae hervorzuheben. Artenzahl, Abundanz und Biomasse sind von Standort zu Standort auf diesem Substrat sehr verschieden. Es handelt sich offenbar um eine Reihe bisher nicht ausreichend zu definierender Teilgemeinschaften einer größeren Zönose. Im Bereich einer Kalkalgenplattform wurde eine 1982 aus ca. 5000 Individuen bestehende Population von Strombus gigas beobachtet (d. h. etwa 1 Tier auf $23 \mathrm{~m}^{2}$; Botero, im Druck). Im März 1983 war der Bestand auf etwa 1 Tier auf $30 \mathrm{~m}^{2}$, im Juli 1983 auf 1 Tier pro $60 \mathrm{~m}^{2}$ zurückgegangen. Ursache für den Rückgang war die Entnahme der Schnecken durch Fischer. Die Gehäuse dieser großen Schnecken werden von Lithophaga aristata, Arca zebra und imbricata sowie von kleinen Barbatia domingensis und Spondylus ictericus besiedelt.

Korallen bilden einen sehr vielseitigen Lebensraum, der von mosaikartig nebeneinander auftretenden Tiergemeinschaften genutzt wird, die hier nur summarisch bearbeitet werden konnten. 32 Muscheln, 2 Käferschnecken und 47 Schnecken wurden festgestellt, von denen einige obligatorisch an Korallen gebunden sind (z. B. Lithophaga antillarum, Pteria colymbus, Calliostoma jujubinum, Trivia nix, quadripunctata, suffusa, pediculus, Simnia acicularis, Cyphoma gibbosum, Bursa thomae, Coralliophila abbreviata, caribaea, scalariformis) (Abb. 10).

Die stets der Luft ausgesetzten Stämme und Stelzwurzeln der Mangrove (Rhizo-

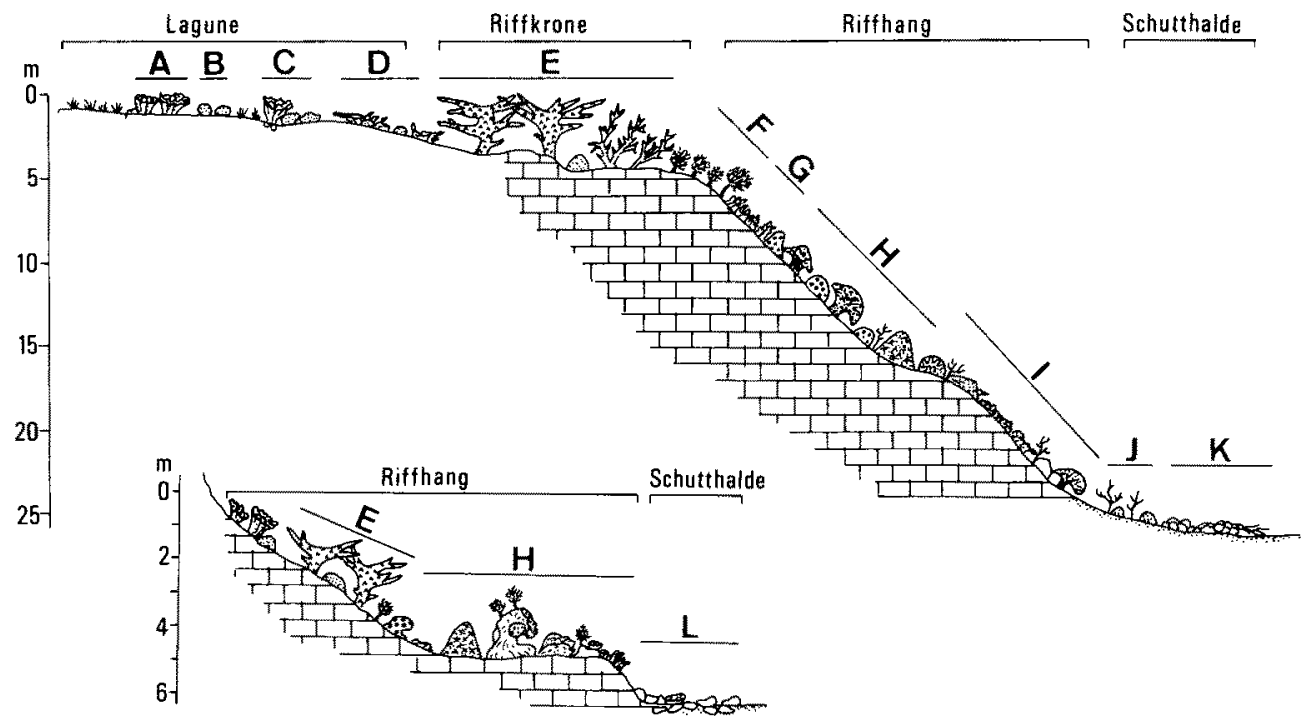

Abb. 10. Vereinfachte Darstellung eines Lagunensaumriffes (oben) und eines Ufersaumriffes (unten). A Feuerkorallen (Millepora spec.) im Flachwasser; B Rollkorallen; C Korallenhorste; D Korallenbruchstücke und -reste; E Acropora-Zone (A. palmata und cervicornis); F Hornkorallen (Gorgonia ventalina u. a.); G Agaricia-Zone mit Grottensystem; $\mathrm{H}$ massiv wachsende Korallen mit Grottensystem; I Mischzone mit massiven und krustenbildenden Korallen; ${ }_{i}$ Riffbasis mit vereinzelten Korallenkolonien; K Korallentrümmer; $\mathrm{L}$ wie $\mathrm{K}$, jedoch weniger tief 
phora mangle) sind ausschließlich von Littorina angulifera besiedelt, die nach Coomans (1969) immer und nur an Mangroven zu finden ist. Die Schnecken bilden Gruppen von 2 bis 7 Tieren, die sich meist dicht über der Wasseroberfläche aufhalten und offensichtlich eine dem Gezeitenrhythmus folgende, kurze, gemeinsame Wanderung durchführen. Ab etwa $40 \mathrm{~cm}$ über dem Wasserspiegel werden die Schnecken seltener, einzelne Individuen sind jedoch auch im Blätterdach in ca. $3 \mathrm{~m}$ Höhe zu finden. In der Höhenverteilung zeigt sich eine signifikante Tendenz $(P<0,05)$ zur Aufteilung nach Größenklassen. An naß bleibenden, gut beschatteten Stellen sind vereinzelt Nerita tessellata, Acmaea antillarum und Chiton tuberculatus nachzuweisen. Artenreicher wird die Gemeinschaft an den ständig vom Wasser bedeckten Teilen der Stelzwurzeln. Die häufigste Muschel ist hier Isognomon bicolor; die Mangroveauster Crassostrea rhizophorae kommt fast ausschließlich in juvenilen Exemplaren (3-6 cm lang) vor. Auch Lopha frons, Brachidontes exustus und Hemitoma octoradiata sind selten. Sehr häufig ist Fissurella angusta, von den vagilen Schnecken treten Nassarius vibex und Cymatium pileare regelmäßig auf.

An gestrandeten Baumstämmen wurde gelegentlich Littorina nebulosa gefunden. $\mathrm{Zu}$ erwähnen sind noch einige endobiontische bzw. parasitische Mollusken. So kommen in Schwämmen Arca imbricata, Lyonsia beana und Vermicularia spirata vor. Von einer etwa $50 \mathrm{~cm}$ langen Seewalze (Astichopus multifidus) wurden 217 Exemplare von Melanella intermedia abgelesen. In Sedimentproben war Cochliolepis parasitica (Tornidae) nachzuweisen, die an Polychaeta leben soll (Moore, 1972).

\section{Strukturvergleich und Identitätsgrad der Malakozönosen}

Die untersuchten Substrattypen unterscheiden sich in ihrer Besiedlung durch Mollusken wesentlich. Besonders deutlich heben sich die Mollusken der Hartsubstrate von denen der Sedimente ab. Das gilt nicht nur auf Art-Niveau, sondern auch für höhere Kategorien. Nur wenige Arten können Sedimente und Hartböden gleichermaßen bewohnen. Das sind grundsätzlich detritus- und pflanzenfressende Mikrogastropoden wie Alaba incerta, Diastoma varium und Finella dubia. Die Abb. 11 zeigt die Verteilung des gesamten, lebenden Artenbestandes der Bahía de Nenguange und die Zuordnung zu den Klassen der Weichtiere.

Die Bivalvia können besonders in der Thalassia-Gemeinschaft sehr stark vertreten sein, stellenweise auch in den Ufersaumzönosen von Felsen, Mangroven (z. B. Isognomon bicolor) und Sandstränden (Donax denticulatus). Im allgemeinen liegt das Verhältnis der Individuenzahlen von Gastropoda und Bivalvia bei 3:1. Eine bemerkenswerte Ausnahme bildet die "Limopsis-Phase" der Grobsand-Gemeinschaft (Station 8), wo das genannte Verhältnis umgekehrt ist und wo allein Limopsis antillarum beinahe $60 \%$ aller Individuen stellt. Eine einzige Art der Scaphopoda (Cadulus quadridentatus) besiedelt fast alle Sedimentböden der Nenguange, ist jedoch niemals dominant: 4,2\% im Schlick, 0,6\% im Feinsand, 0,2\% im Mischsand und in Thalassia-Wiesen, 0,7\% im Grobsand.

Trotz aller Schwierigkeiten bei der Ermittlung lassen sich für die Biomassewerte einige allgemeinere Aussagen machen. Die höchsten Werte wurden im Eulitoral des Steingeröllstrandes festgestellt $\left(359 \mathrm{~g} / \mathrm{m}^{2}\right.$, Feuchtgewicht), aus methodisch-praktischen Gründen bezogen auf einen dreidimensionalen "Geröllquadratmeter". Sedimente mit 


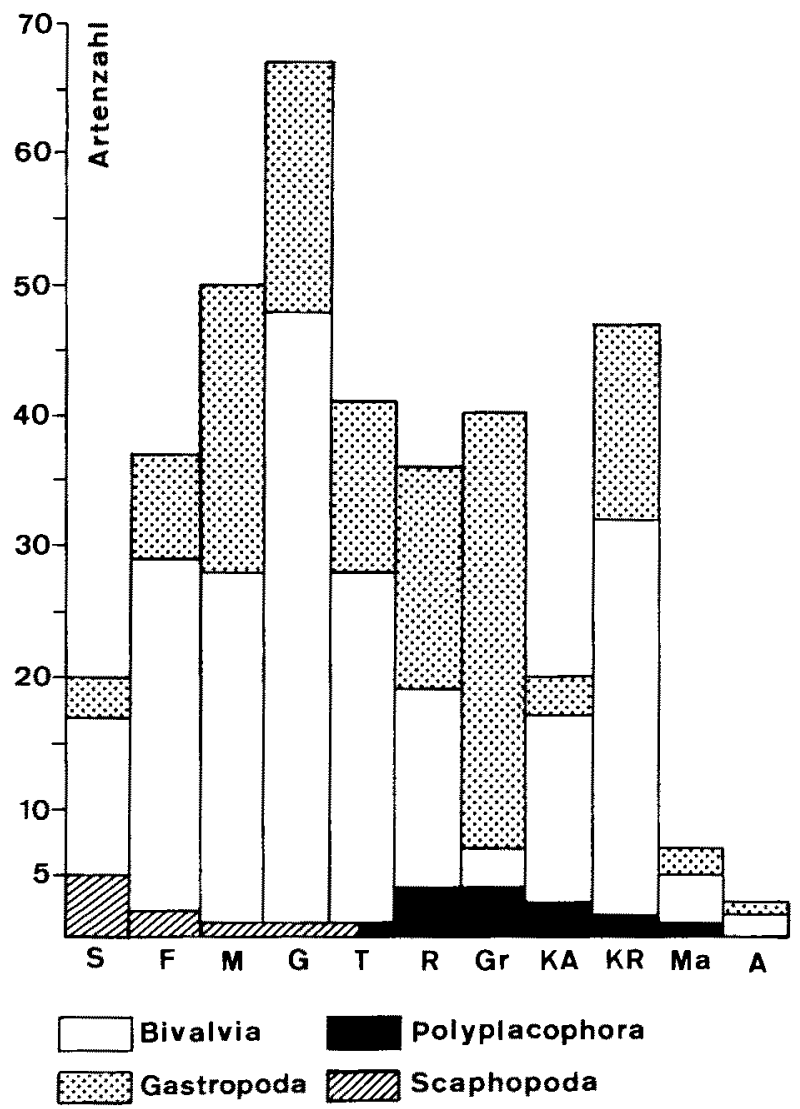

Abb. 11. Artenzahl und Anteil der Molluskenklassen in den untersuchten Substrattypen. S Schlick, F Feinsand, M Mischsand, G Grobsand, T Thalassia, R Felsufer, Gr Geröllstrand, KA Kalkalgengrund, KR Korallenriffe, Ma Mangrove, A sonstige Substrate

Thalassia-Bewuchs gaben Durchschnittswerte von $142 \mathrm{~g} / \mathrm{m}^{2}$ bis in $1 \mathrm{~m}$ Tiefe und $69 \mathrm{~g} / \mathrm{m}^{2}$ zwischen 1 und $5 \mathrm{~m}$. In seegrasfreien Sedimenten des Flachwassers ist die MolluskenBiomasse gering (im Mittel $2,2 \mathrm{~g} / \mathrm{m}^{2}$ ).

Die Gastropoden dominieren in und auf allen Substraten, Scaphopoda bewohnen ausschließlich Weich-, Polyplacophora nur Hartsubstrate. Zwar ist die höchste Artenzahl auf Grobsand nachzuweisen (115 Spezies), doch ist die Artenzahl pro Flächeneinheit auf Mischsand höher, nämlich 60,5 gegenüber 39,7 Arten/0,25 $\mathrm{m}^{2}$. Die Anzahl der Individuen ist in und auf den bei 15-16 m Tiefe gelegenen Misch- und Grobsandböden am höchsten, darunter sind allerdings viele kleine Formen (durchschnittlich 3660 Individuen $/ \mathrm{m}^{2}$ bei Misch-, 3200 bei Grobsandböden). In litoralen Felstümpeln kann die Wohndichte einiger weniger Arten auf 8000-12 000 Tiere ansteigen (Littorina meleagris und mespillum, Petaloconchus varians). Ausgesprochen individuenarm sind einige Fein- und Mischsande vor Playa Oriental und Playa Brava sowie die Ufersaumzönosen im Eulitoral aller Sandstrände (890 bzw. 332 bzw. 0-30 Individuen $/ \mathrm{m}^{2}$ ). Im Schlick liegt 
die Siedlungsdichte bei durchschnittlich 1050 Tieren $/ \mathrm{m}^{2}$, kann aber an einzelnen Stationen (z.B. 3) auch nur die Hälfte betragen. Im Mischalgenbereich des Felsufers wurden zwischen 7 und 16 Arten nachgewiesen, mit durchschnittlich 244 Individuen/ $\mathrm{m}^{2}$. Die Biomassewerte an wenig exponierten Stellen sind niedriger $\left(22 \mathrm{~g} / \mathrm{m}^{2}\right)$ als an solchen starker Brandung $\left(31 \mathrm{~g} / \mathrm{m}^{2}\right)$. An letzteren treten größere Arten (wie Cittarium pica, Purpura patula, Acanthopleura granulata) häufiger auf als an ruhigeren Küstenabschnitten, Arten- und Individuenzahl insgesamt sind hier dagegen geringer.

Die Arten-Individuen-Relationen sind in zeitlich und räumlich etablierten Beständen von Zönose zu Zönose so verschieden, daß sie zur Charakterisierung herangezogen werden können. Aus Abb. 12 sind die durchschnittlichen Werte für die hier untersuchten Molluskenzönosen der Sedimentböden zu entnehmen. Es ist auch deutlich, daß die Abweichungen z. T. beträchtlich sind, vor allem in grobsandigen Sedimenten.

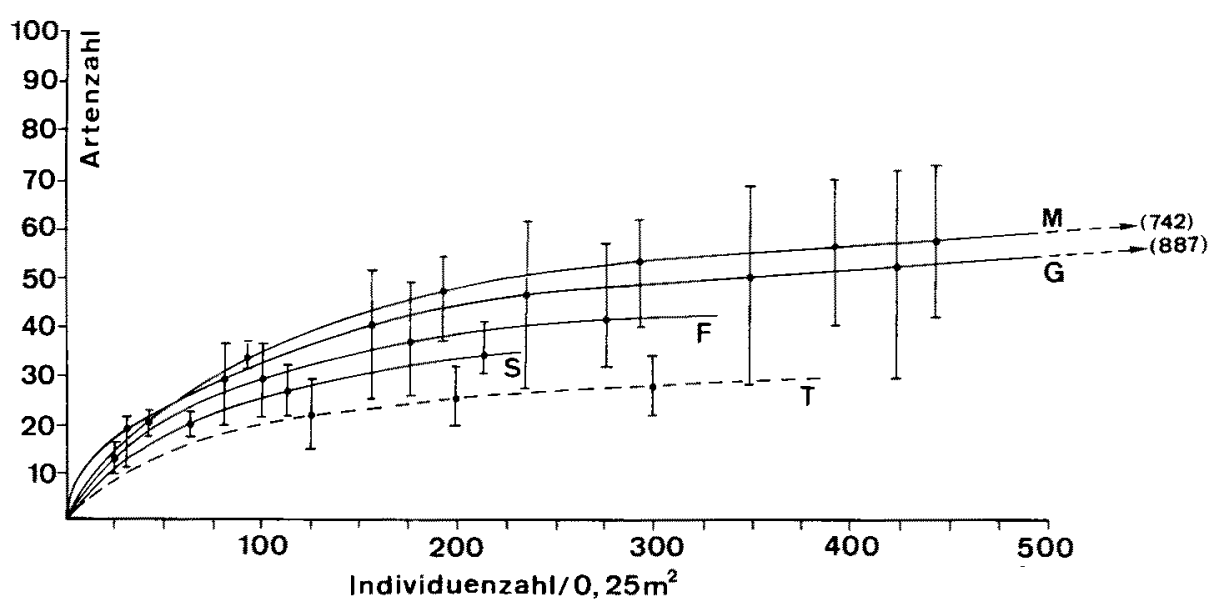

Abb. 12. Durchschnittliche Relationen von Individuen- und Artenzahlen der Malakozönosen in und auf typischen Substraten (mit Standardabweichungen). Abkürzungen wie in Abb. 11

Die höchste Diversität wurde für eine Station (4) ermittelt, die auf Grobsand in $23 \mathrm{~m}$ Tiefe liegt. Hier waren 88 Arten mit 1310 Individuen vertreten (auf $0,25 \mathrm{~m}^{2}$ ). Ihr Diversitätswert $\mathrm{H}^{\prime}$ lag mit 3,525 wesentlich über dem für Grobsande in der Nenguange berechneten Durchschnitt von 2,816. Alle anderen Sedimente weisen höhere Werte auf: Mischsand 3,332, Feinsand 3,001, Schlick 3,070 und Seegraswiesen 2,783. Setzt man die Diversität in Bezug zur Artenzahl und zur Äquität, so zeigt sich, daß bei den Zönosen von Feinsand und in der Seegraswiese Veränderungen der Diversität im wesentlichen durch die Änderung der Artenzahl bedingt sind, während in Grobsand die Äquität die ausschlaggebende Rolle spielt. Vom Sandboden der Uferzone erhöhen sich mit zunehmender Tiefe die Arten- und Individuenzahlen, die Biomasse und die Diversität. Einen auffälligen Höhepunkt erreichen diese Werte vor der Playa Oriental in 3-4 m Tiefe. Der Feinsand weist an dieser Stelle einen besonders hohen Anteil an Schlamm auf und ist stellenweise mit Thalassia bedeckt. So kommen hier besonders viele detritivore Arten und Seegrasbewohner vor, wie z. B. Olivella minuta, Terebra weisbordi, Drillia minor, Pyrgocythara filosa, Bulla striata, Nassarius consensus. Am Felsufer wechselt die Diver- 
sität sehr stark, was mit der räumlich eng begrenzten Ausdehnung der Ufersaumzönosen dieses Bereichs korrespondiert. Vom Supralitoral abwärts nimmt die Diversität von 0 auf 1,5 im eulitoralen Mischalgenbereich und 2,1 im oberen Sublitoral des Geröllstrandes zu.

Die Dominanz spiegelt in besonderem Maße die strukturellen und funktionellen Beziehungen in den Zönosen wider. Wird die Äquität als Maßzahl genommen und gegen die Artenzahl aufgetragen, so liegen die Punkte in bestimmten Feldern benachbart. Werden die Extremwerte für die einzelnen Substrattypen miteinander verbunden, so ergibt sich das übersichtlichere Bild der Abb. 13. Deutlich wird daraus, daß es zwischen den Molluskengemeinschaften der verschiedenen Substrate zwar zahlreiche Uberlappungen gibt, doch sind zwei Gruppen klar voneinander getrennt: die Zönosen des Ufersaumes von denen der sublitoralen Sedimente. Bei letzteren ist trotz der Überlappungen festzustellen, daß die Äquität vom Schlick zum Grobsand hin geringer wird.

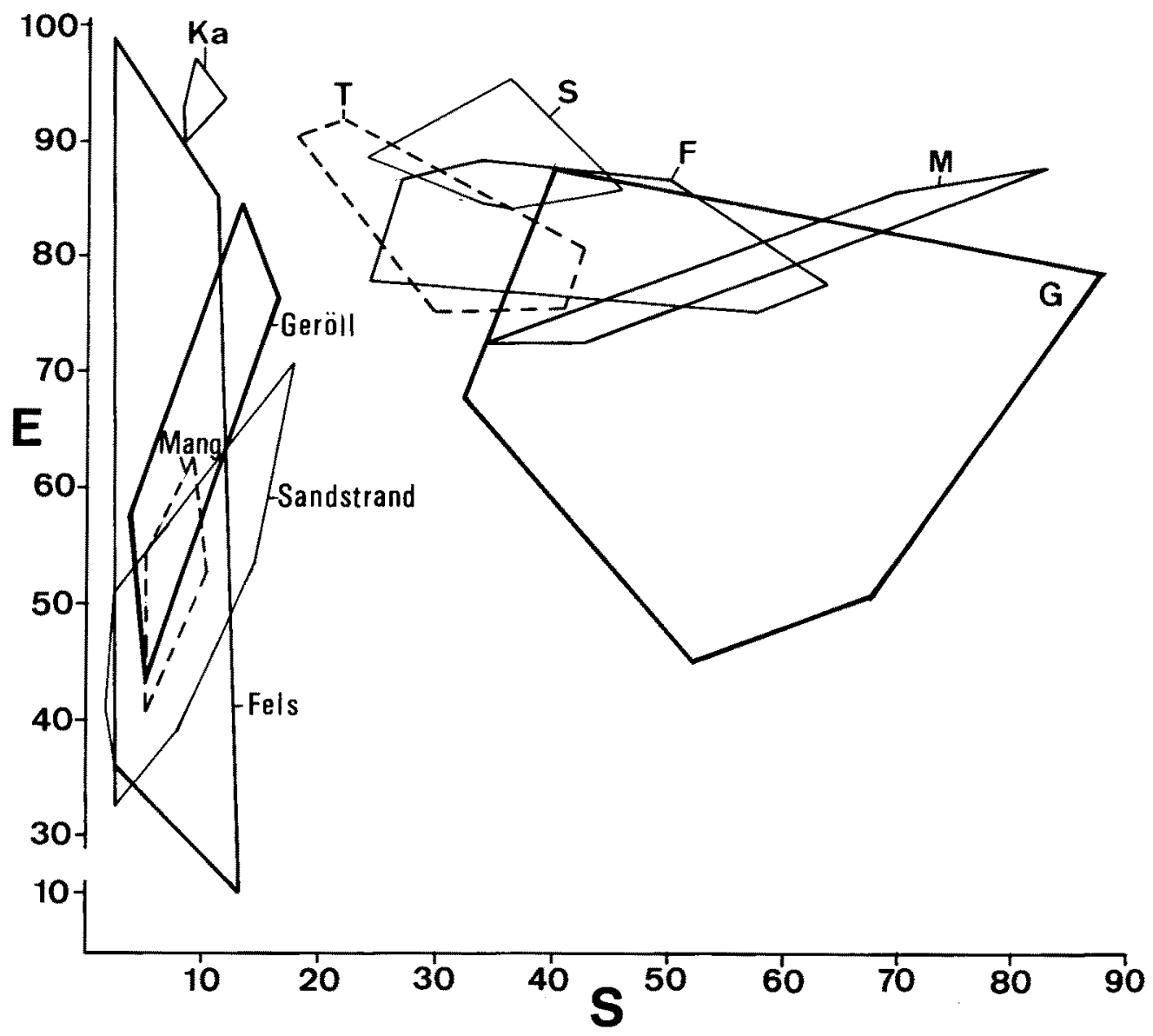

Abb. 13. Vielfältigkeits-Diagramm der Malakozönosen verschiedener Substrattypen. E evenness, $S$ Artenzahl. Fels: Felsufer, Geröll: Geröllstrand, Mang: Mangrove. Ubrige Abkürzungen wie in Abb. 11 
Ausgehend vom Ahnlichkeitsquotienten nach Bray-Curtis wurde die Identitätsfolge in Form eines Dendrogramms ermittelt (Abb. 14). Legt man als kritische Grenze $30 \%$ Ähnlichkeit zugrunde, so bekommt man eine Gruppierung der Stationen, die der Verteilung der Substrattypen entspricht (Ausnahme: Station 31). Besonders groß sind die Übereinstimmungen der Molluskenbesiedlung auf Grob- und Mischsandböden. Andererseits gibt es einige Stationen (z. B. 13, 13-3,4;24-D, E), die verschiedene Hauptgruppen verknüpfen, was aus dem zweidimensionalen Dendrogramm nicht zu

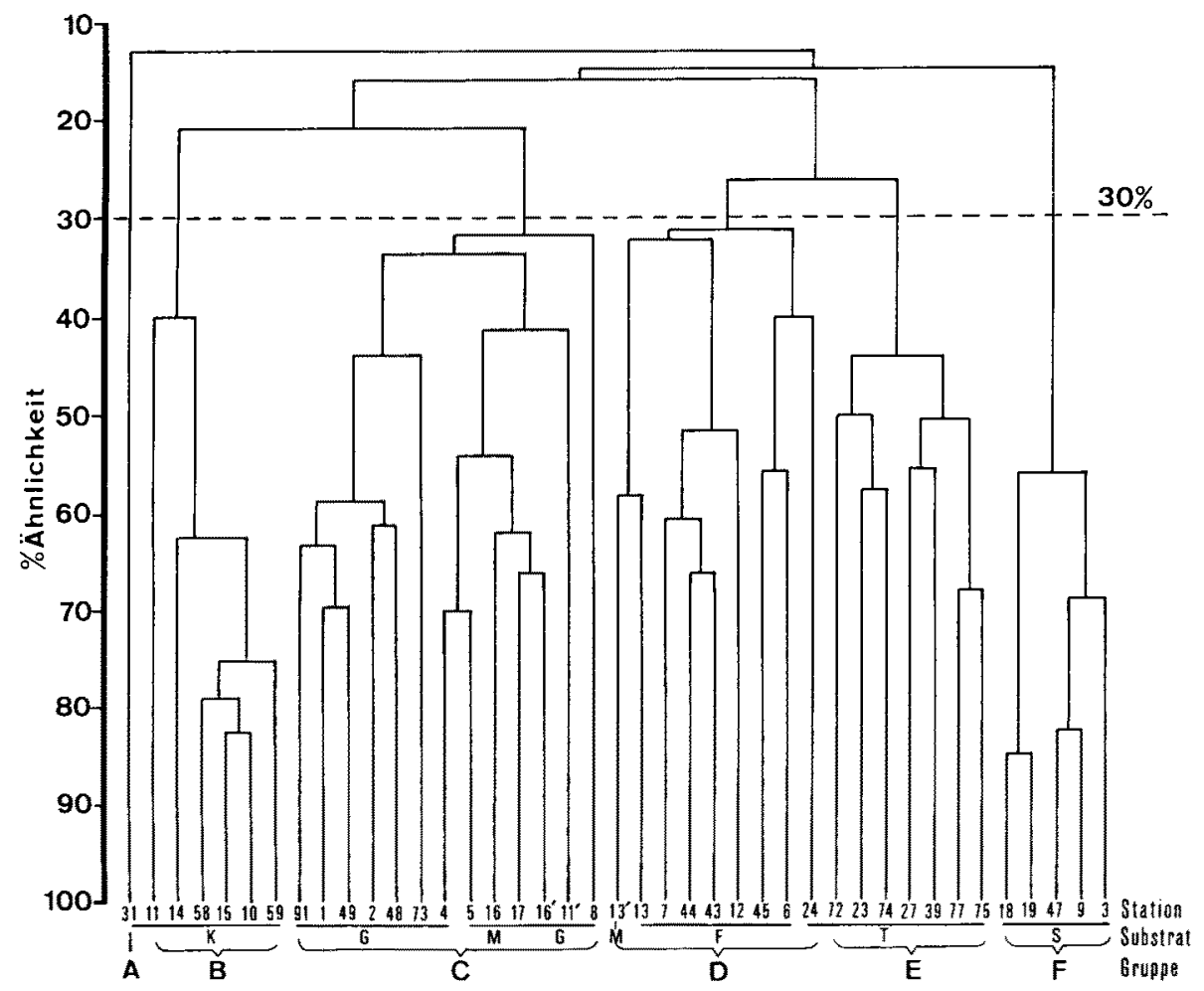

Abb. 14. Ahnlichkeits-Dendrogramm zur Charakterisierung und Gruppierung der Molluskenbestände in und auf bestimmten Substrattypen. Abkürzungen wie in Abb. 11. Nähere Erläuterung im Text

entnehmen ist; diese Beziehungen könnten nur durch ein räumliches Modell veranschaulicht werden. In allen Fällen liegt die Ähnlichkeit der untersuchten Molluskengemeinschaften bei mehr als $10 \%$ (Abb. 14), wofür eine Reihe ubiquitärer Arten verantwortlich ist, die vom Flachwasser bis in größere Tiefen und auf verschiedenen Substrattypen vorkommen. Manche dieser Arten sind eudominant. Zur Berechnung von Nischenbreite und -überlappung wurde das Substrat nach der Korngröße in 7 Klassen eingeteilt (bis 0,$066 ; 0,066-0,125 ; 0,125-0,25 ; 0,25-0,5 ; 0,5-1,0 ; 1,0-2,0$; über $2,0 \mathrm{~mm}$ Korndurchmesser). Die Nischenbreiten für einige ausgewählte Arten sind der Tabelle 1 zu entnehmen. Besonders hohe Werte ergeben sich für je 3 Bivalvia und Gastropoda. Oft 
Tab. 1. Indices der Nischenbreite einiger ubiquitärer Arten

\begin{tabular}{|c|c|c|}
\hline Taxa & Species & Index \\
\hline Bivalvia & $\begin{array}{l}\text { Gouldia cerina } \\
\text { Crassinella cf. martinicensis } \\
\text { Ervilia nitens } \\
\text { Nucula venezuelana } \\
\text { Crassinella guadelupensis }\end{array}$ & $\begin{array}{l}3,43 \\
3,40 \\
3,11 \\
1,55 \\
1,53\end{array}$ \\
\hline Gastropoda & $\begin{array}{l}\text { Caecum floridanum } \\
\text { Diastoma varium } \\
\text { Olivella perplexa } \\
\text { Alaba incerta } \\
\text { Finella dubia } \\
\text { Acteocina candei } \\
\text { Marginella eburneola } \\
\text { Caecum regulare } \\
\text { Caecum cornucopiae } \\
\text { Caecum imbricatum } \\
\text { Persicula lavaellana } \\
\text { Granulina ovuliformis }\end{array}$ & $\begin{array}{l}3,79 \\
3,48 \\
3,07 \\
2,85 \\
2,39 \\
2,22 \\
2,05 \\
2,04 \\
1,95 \\
1,83 \\
1,55 \\
1,41\end{array}$ \\
\hline
\end{tabular}

ist eine direkte Beziehung zwischen Nischenbreite und -überlappung erkennbar. Die Arten mit der geringsten Nischenbreite, nämlich Granulina ovuliformis und Crassinella guadelupensis, weisen auch die kleinste Nischenüberlappung (Tab. 2) auf. Größte Nischenüberlappung besteht entsprechend zwischen den beiden Bivalvia mit der größten Nischenbreite (Crassinella of. martinicensis, Gouldia cerina). Caecum floridanum,

Tab. 2. Nischenüberlappung einiger ubiquitärer Mollusken der Bahía de Nenguange (die Arten sind durch in Klammern gestellte Ziffern gekennzeichnet)

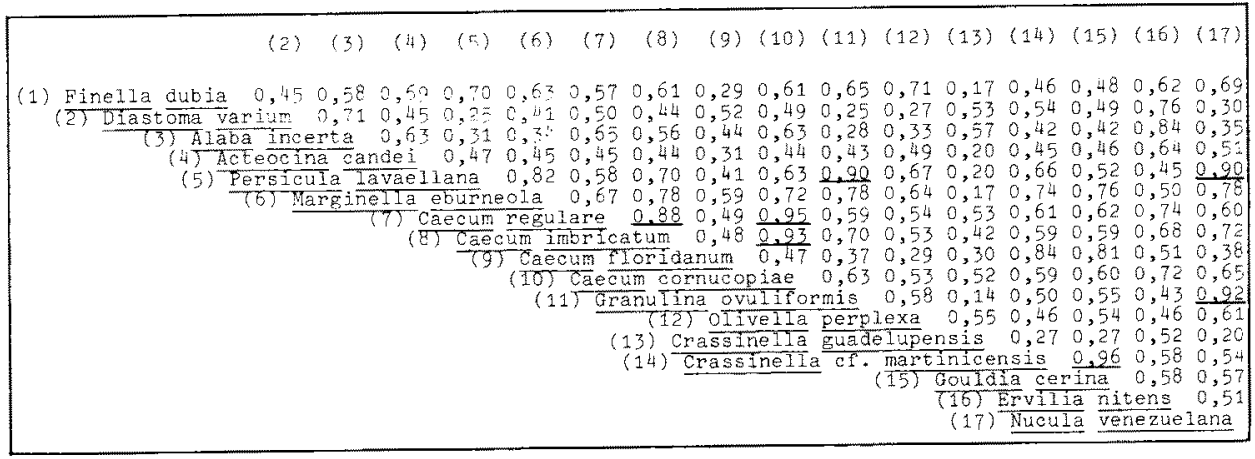

die Art mit der größten Nischenbreite, zeigt nur geringe Überlappung mit den anderen Arten derselben Gattung. Die Schnecken Finella dubia, Diastoma varium, Alaba incerta und Olivella perplexa sind epifaunische Formen, die vermutlich geringe Ansprüche an das Substrat stellen. 


\section{DISKUSSION}

Die Bahía de Nenguange ist in ökologischer Hinsicht ein für die Region von Santa Marta und den Tayrona-Nationalpark repräsentatives Gebiet. In dieser überschaubar kleinen Bucht sind aufgrund der physiographischen Voraussetzungen alle für das Gebiet typischen marinen Habitate vorhanden, was in der besonders artenreichen Molluskenfauna zum Ausdruck kommt. Brackwasser- bzw. Ästuargebiete und damit zusammenhängend Mangrovebestände sind allerdings hier nur spärlich ausgebildet; sie sind in den benachbarten Buchten Chengue und Cinto vielseitiger entwickelt, da dort die Süßwasserzufuhr durch größere Bäche das ganze Jahr über gewährleistet ist. Ein eingehender Vergleich mit ähnlichen Buchten der karibischen Region, auch speziell der kolumbianischen Küste, ist z. Z. nicht durchzuführen, da benthos-ökologische und hydrographische Informationen nur in beschränktem Umfang vorliegen. Vergleichbar wäre von Größe, Topographie und Beschaffenheit der Substrate am ehesten die Scotland Bay (Chaguaramas, Trinidad; Alkins \& Kenny, 1980), wenn die Untersuchungsmethoden und die Technik der Auswertung abgestimmt würden. Besonders hervorzuheben ist der Artenreichtum der Nenguange. Zu den bisher an der Karibikküste Kolumbiens nachgewiesenen Mollusken (u. a. Dautzenberg, 1900; Daniel, 1941; Kaufmann \& Götting, 1970; Bayer, 1971; Cosel, 1973, 1978; Götting, 1973; Bandel, 1974, 1976a, b, c, 1977; Marcus, 1976; Duque, 1979) kommen mindestens weitere 78 hinzu: 60 Gastropoda, 14 Bivalvia, 1 Scaphopoda, 3 Polyplacophora. Am ausführlichsten untersucht wurde im Gebiet bisher die ca. $50 \mathrm{~km}$ südwestlich der Nenguange gelegene Ciénaga Grande de Santa Marta (Cosel, 1978), ein typisches Brackwasserhaff, das sich geomorphologisch-hydrographisch und entsprechend auch faunistisch-ökologisch wesentlich von der Nenguange unterscheidet. Der Boden der flachen, maximal $5 \mathrm{~m}$ tiefen Ciénaga besteht zum großen Teil aus Schlamm mit unregelmäßig verteilten Schill-Ansammlungen. Die Molluskenzönosen werden nicht nur vom Substrat, sondern ganz wesentlich auch von der räumlich und jahreszeitlich schwankenden Salinität bestimmt. Unter den Mollusken dominieren dort für mixo- bzw. polymixohaline Bereiche charakteristische Arten. So sind von den 98 im Ciénaga-Becken nachgewiesenen, lebenden Molluskenarten nur 37 auch in der Nenguange zu finden, die meisten von ihnen auf Hartsubstrat der Gezeitenzone.

Ein ca. $4 \mathrm{~km}^{2}$ großes Gebiet um die Insel Payardi (Panamá) weist neben verschiedenen Sedimenttypen auch Korallenriffe und Seegraswiesen auf (Radwin, 1969). Hier wurden insgesamt 297 Molluskenarten nachgewiesen, von denen 167 (=56\%) auch in der Nenguange vorkommen. Nähere Angaben zur Hydrographie liegen nicht vor. In einem ca. $7 \mathrm{~km}^{2}$ großen, halbgeschlossenen, bis $4 \mathrm{~m}$ tiefen Haff bei Playa de Vega Baja (Puerto Rico) ermittelten Arnow et al. (1963) eine durchschnittliche Wassertemperatur von $26^{\circ} \mathrm{C}$ und eine Salinität von $37 \%$. Auf Sand, Fels und erratischen Blöcken wurden 224 Mollusken nachgewiesen, von denen 194 (= 87\%) auch in der Nenguange vorkommen. Olsson \& McGinty (1958) beschrieben für die etwa $78 \mathrm{~km}^{2}$ große Insel Bocas (Panamá) im Verlaufe von 30 Jahren 467 Arten (davon sind $239=51 \%$ in der Nenguange anzutreffen). Coomans (1963) führt für die wenig größere Insel Saint Martin (Kleine Antillen) 343 Arten an, von denen $220(=63 \%$ ) mit solchen aus der Nenguange identisch sind. Dagegen werden für Grenada $\left(344 \mathrm{~km}^{2}\right)$ nur 171 Mollusken gemeldet (Hemmen \& Hemmen, 1979), für Grand Cayman 293 (Abbott, 1958), für St. Croix 712 (Nowell-Usticke, 1959), für Puerto Rico 840 (Humfrey, 1975), von Yucatán 769 (Vokes \& 
Vokes, 1983) und aus Surinam 271 (Altena, 1971, 1975). In allen diesen Fällen ist aufgrund fehlender ökologischer Daten der Vergleich mit den Verhältnissen in der Nenguange nicht möglich. Auf die interessanten zoogeographischen Aspekte wird an anderer Stelle eingegangen (Diaz \& Götting, 1987).

Die Molluskenbesiedlung am Felsufer der Nenguange weist eine deutliche Zonierung auf, wie sie auch außerhalb der Bucht an der Felsküste zu beobachten ist. Die Anordnung der Mollusken zeigt Ubereinstimmungen mit der von Brattström (1980) dargestellten Zonierung zahlreicher Algen und anderer Tierarten. Weniger stark gegliedert sind die Ufersaumzönosen auf Sandböden, die anhand ihrer Molluskenfauna in 3 Zonen einzuteilen sind, gekennzeichnet durch Donax denticulatus im oberen Eulitoral, Terebra cinerea in der Brandungszone und Mazatlania aciculata in der unteren Brandungszone und dem oberen Sublitoral. Die Wohndichte dieser Arten und die Ausdehnung der von ihnen besiedelten Bereiche variieren entlang der Küste stark. In Playa Brava kommt $M$. aciculata in der unteren Brandungszone massenhaft vor; $T$. cinerea dringt bis in den wassergesättigten Eulitoralbereich vor. In Playa Oriental dagegen konzentriert sich $T$. cinerea ausschließlich in der unteren Brandungszone, wo $M$. aciculata fehlt. Diese letztere Situation entspricht ungefähr der typischen Zonierung an Stränden, an denen Donax denticulatus und $T$. cinerea, nicht aber $M$. aciculata vorhanden sind, z. B. in Jamaica (Wade, 1967) und bei Cartagena, Colombia (Dexter, 1973). $T$. cinerea ernährt sich zum Teil von Donax, $M$. aciculata möglicherweise auch. So könnte die räumliche Verteilung beider Arten auf Nahrungs- und/oder Raumkonkurrenz zurückzuführen sein. Ein anderer, häufiger Bewohner karibischer Sandstrände, Tivela mactroides (Rodríguez, 1959; Dexter, 1973), ist in der Nenguange und der benachbarten Region nicht vorhanden. Auch Heterodonax bimaculatus, sonst oft im Eulitoral mit Donax denticulatus vergesellschaftet, findet sich in der Nenguange nur stellenweise im kiesig-grobsandigen Strand von Playa Viva.

Die Mollusken der Mangrove bilden in der Nenguange eine verarmte typische Zönose dieses Lebensraumes. Von den 19 von Coomans (1969) genannten Arten, die immer an Mangrovenwurzeln zu finden sein sollen, gibt es hier nur 6 . Hinzu kommen in der Bucht weitere 6 Spezies, die die Mangrove alternativ besiedeln (vor allem Nerita tessellata, Acmaea antillarum und Nassarius vibex). Chiton tuberculatus, sonst auf primärem Hartsubstrat zu finden, lebt in der Nenguange nur an Mangrovenwurzeln.

Ubber die Mollusken der Korallenriffe können nur qualitative Aussagen gemacht werden, doch sind für einige Arten Bindungen an bestimmte Riffstrukturen erkennbar. Als besonders artenreich erwiesen sich Riffzonen mit vorwiegend massiven Korallen und Grottensystemen. Bereiche mit akkumulierten Korallentrümmern und Korallenresten bis ca. $8 \mathrm{~m}$ Tiefe beherbergen ebenfalls zahlreiche Mollusken; unter $8 \mathrm{~m}$ nehmen Arten- und Individuenreichtum ab. Auch an der Riffkrone, wo Acropora-Arten vorherrschen, leben wenige Mollusken. Von 7 Arten, die Hadfield (1976) als mit lebenden Korallen assoziiert bezeichnet, kommen 5 in der Nenguange vor (Cyphoma gibbosum, Simnia acicularis, Coralliophila abbreviata und caribaea, Pteria colymbus, Lithophaga antillarum). Während $P$. colymbus und $L$. antillarum Korallen nur als Substrat benutzen, ernähren sich die anderen von Korallen (C. gibbosum und $S$. acicularis ausschließlich von Gorgonien).

Unter den von uns nachgewiesenen Gastropoda gibt es weitere 7 Arten, die ausschließlich auf und unter Korallen gefunden wurden und vermutlich eng an diese 
gebunden sind: Calliostoma jujubinum, 4 Trivia-Arten, Bursa thomae und Coralliophila scalariformis. Bis auf $B$. thomae haben alle diese Arten coralliophage Verwandte in der Karibik bzw. im Indopazifik (vgl. Robertson, 1970; Hadfield, 1976). B. thomae wurde auf einer Kolonie von Dichocoenia spec. gefunden, inmitten einer ca. $15 \mathrm{~cm}^{2}$ großen, fleckig ausgebleichten Fläche, die den Fraßspuren anderer Schnecken glich.

Für die sublitoralen Zönosen der Sedimentböden und der Seegraswiesen wurde eine starke Korrelation zwischen den auf Substratmerkmalen basierenden und den aus der zönologischen Analyse (Bray-Curtis-Index, Dendrogramm) resultierenden Gemeinschaften festgestellt. Dendrogramme können nur eine unzureichende Hilfe zur Veranschaulichung zönologischer Informationen sein: sie erzwingen meist künstlich geschaffene Gruppierungen. Kontinua zwischen den Zönosen werden oft verdeckt. Gerade die höhere Komplexität ökologischer Systeme in den Tropen macht es schwierig, "klassische" Zönosen im Sinne von Petersen (1915; vgl. auch Gray, 1981) abzugrenzen. In kalten und gemäßigten Regionen haben Benthos-Gemeinschaften infolge hoher Konstanz und Treue vieler Arten in verschiedenen Teilen der Weltmeere oft verblüffende Ähnlichkeit (Parallelgemeinschaften; Thorson, 1971), da in ihnen zwar verschiedene Arten, aber diese aus denselben Gattungen vorkommen. "Petersen-Zönosen" sind auch aus einigen Teilen warmer Meere bekanntgeworden (Westafrika: Buchanan, 1958; Indien: Seshappa, 1953). In der Nenguange weist lediglich die Gemeinschaft der Seegraswiesen "Charakterarten" auf, die immer und nur in ihr auftreten (Codakia- und Lucina-Arten). Die übrigen Zönosen werden besser nach den Substrattypen gekennzeichnet.

Für die Bahía de Nenguange ergeben sich aufgrund der vorliegenden Untersuchungen folgende 12 Malakozönosen:

(1) Die Ufersaumzönose auf Felsen: eine in 5-6 Zonen gegliederte Zönose zwischen dem oberen Sublitoral und dem Supralitoral, die je nach Böschungsgrad und Wellenexposition unterschiedlich breit ist.

(2) Die Ufersaumzönose des Steingeröllstrandes: sie bildet sich an geschützten Stellen zwischen dem oberen Sublitoral und dem oberen Eulitoral; rascher Artenwechsel.

(3) Die Ufersaumzönose der Mangrove: eine durch die Wasseroberfläche in zwei Bereiche unterteilte Gemeinschaft an Stelzwurzeln und Stämmen von Rhizophora mangle.

(4) Die Ufersaumzönose des Sandstrandes.

(5) Die Thalassia-Zönose: auf verschiedenen Sedimenten, die von Thalassia testudinum bewachsen sind; sie erstreckt sich vom Flachwasser bis in ca. $10 \mathrm{~m}$ Tiefe.

(6) Die Schlammsand-Feinsand-Zönose: vom Flachwasser bis ca. $4 \mathrm{~m}$ Tiefe; sie kann als Teil der Feinsand-Mischsand-Zönose angesehen werden.

(7) Die Feinsand-Mischsand-Zönose: vom Flachwasser bis ca. $20 \mathrm{~m}$ Tiefe.

(8) Die Grobsand-Zönose: überwiegend in Korallensand in der Nähe von Riffbauten; vom Flachwasser bis ca. $6 \mathrm{~m}$ Tiefe.

(9) Die Mischsand-Grobsand-Schill-Zönose: mosaikartig verteilt, ab $8 \mathrm{~m}$ Tiefe; mit erheblichen Uberlappungen mit der Feinsand-Mischsand- und der KalkalgengrundZönose; in ihr sind zwei Hauptphasen zu unterscheiden: in der einen dominieren Carditopsis smithii und Caecum spp., in der anderen herrscht Limopsis antillensis vor.

(10) Die Schlick-Zönose: beschränkt auf die tiefen Lagen der Senken ab ca. 26 m; 
das Substrat ist grauer Schlick bzw. Schlicksand mit hohem Anteil an organischem Material.

(11) Die Kalkalgengrund-Zönose: in der Westhälfte der Nenguange in 10-15 m Tiefe; mosaikartig verteiltes Kalkalgengeröll auf Grobsand und Schill.

(12) Die Korallenriff-Zönose: eine je nach Riffbereich und Riffstruktur stark gegliederte Gemeinschaft; vom Flachwasser bis ca. $26 \mathrm{~m}$ Tiefe.

Die Malakozönosen der sublitoralen Sedimente überlappen sich zum Teil beträchtlich, bedingt durch zahlreiche ubiquitäre Arten.

Die vorliegenden, zeitlich begrenzten Untersuchungen spiegeln einen bestimmten Status wider. Sie ermöglichen weder eine Aussage über eine eventuelle Jahresrhythmik noch über die langfristige Dynamik in den Molluskengemeinschaften der Nenguange. Als Hinweise auf solche Veränderungen können Massenfunde frischer Leerschalen einiger Muscheln (Trachycardium isocardia im Dezember, Tellina listeri im Januar/ Februar) und das Auftreten von Gelegen bestimmter Schnecken gedeutet werden (in der Regenzeit im Juni/Juli: Conus ermineus und mappa granarius, Turbinella angulata, Chicoreus brevifrons).

\section{LITERATUR}

Abbott, R. T., 1958. The marine mollusks of Grand Cayman Island, British West Indies. - Monogr. Acad. nat. Sci. Philad. 11, 1-138.

Alkins, M. E. \& Kenny, J. S., 1980. A baseline survey of Scotland Bay, Chaguaramas. - Occ. Pap. Inst. mar. Affairs, Trinidad and Tobago 80/01, 1-68.

Almeida, P., 1974. Distribución de los moluscos en la costa centro-occidental (Patanemo-Punta Tucatas) de Venezuela, comparación de los habitats litorales. - Mems Soc. Cienc. nat. La Salle $34,25-52$.

Altena, C. O. van Regteren, 1968. The holocene and recent marine bivalve Mollusca of Surinam.Stud. Fauna Suriname 10, 153-179.

Altena, C. O. van Regteren, 1971. The marine Mollusca of Suriname (Dutch Guiana), holocene and recent. II: Bivalvia and Scaphopoda. - Zool. Verh. 119, 1-100.

Altena, C. O. van Regteren, 1975. The marine Mollusca of Suriname (Dutch Guiana), holocene and recent. III: Gastropoda and Cephalopoda. - Zool. Verh. 139, 1-104.

Arnow, L., St. Clair, F. \& Arnow, T., 1963. The Mollusca of a lagoonal area at Playa de Vega Baja, Puerto Rico. - Caribb. J. Sci. 3, 163-172.

Bandel, K., 1974. Studies on Littorinidae from the Atlantic. - Veliger 17, 92-114.

Bandel, K., 1976a. Observations on spawn, embryonic development and ecology of some Caribbean lower Mesogastropoda. - Veliger 18, 249-271.

Bandel, K., 1976b. Morphologie der Gelege und ökologische Beobachtungen an Muriciden (Gastropoda) aus der südlichen Karibischen See. - Verh. naturforsch. Ges. Basel 85, 1-32.

Bandel, K., 1976c. Morphologie der Gelege und ökologische Beobachtungen an Buccinaceen (Gastropoda) aus der südlichen Karibischen See. - Bonn. zool. Beitr. 27, 98-133.

Bandel, K., 1977. Spawning, development and ecology of some higher Neogastropoda from the Caribbean Sea of Colombia (South America). - Veliger 19, 176-193.

Bayer, F. M., 1971. New and unusual mollusks collected by the R/V John Elliott Pillsbury and R/V Gerda in the tropical western Atlantic. - Bull. mar. Sci. 21, 111-236.

Brattström, H., 1980. Rocky-shore zonation in the Santa Marta area, Colombia. - Sarsia 65, 163-226.

Buchanan, J. B., 1958. Bottom fauna communities across the continental shelf of Accra, Ghana. Proc. zool. Soc. Lond. 130, 1-56.

Bula, G., 1977. Algas marinas bénticas indicadoras de un área afectada por aguas de surgencia frente a la costa caribe de Colombia. - An. Inst. Invest. mar. Punta Betín 9, 45-71.

Caycedo, I. E., 1977. Fitoplancton de la Bahía de Nenguange (Parque Nacional Tayrona), Mar Caribe, Colombia. - An. Inst. Invest. mar., Punta Betin 9, 17-44. 
Colwell, R. K. \& Futuyima, D. J., 1971. On the measurement of niche breadth and niche overlap. Ecology 52, 567-576.

Coomans, H. E., 1963. The marine Mollusca of Saint Martin, Lesser Antilles, specially from the French part. - J. Conch. 103, 42-111.

Coomans, H. E., 1969. Biological aspects of mangrove mollusks in the West Indies. - Malacologia 9 , 79-84.

Cosel, R. von, 1973. Lista preliminar de los moluscos de la Ciénaga Grande de Santa Marta (Colombia). - Mitt. Inst. colombo-alemán Invest. cient. Punta de Betín. 7, 47-56.

Cosel, R. von, 1978. Die Mollusken der Ciénaga Grande de Santa Marta (Kolumbien) und ihre Lebensgemeinschaften im Wechsel der Jahreszeiten. Diss., Univ. Giessen, 348 pp.

Daniel, H., 1941. Apuntes sobre algunos moluscos colombianos. - Revta Acad. colomb. Cienc. exact. fís. nat. $4,372-380$.

Dautzenberg, P., 1900. Croisères du yacht "Chazalie" dans l'Atlantique. Mollusques. - Mém. Soc. zool. Fr. 13, 9-10.

Dexter, D. M., 1973. Sandy-beach fauna of the Pacific and Atlantic coasts of Costa Rica and Colombia. - Revta Biol. trop. 22, 51-66.

Diaz, J. M. \& Götting, K.-J., 1987. Die Mollusken der Bahía den Nenguange (Karibik, Kolumbien) und ihre zoogeographischen Beziehungen. - Zool. Jb. (Syst. Okol. Geogr. Tiere.) 114 (Im Druck).

Duque, F., 1979. Malacofauna de aguas someras y substrato rocoso en la Bahia de Nenguange. Boln Mus. mar. Bogotá 9, 3-29.

Field, J. G., Clarke, K. R. \& Warwick, R. M., 1982. A practical strategy for analysing multi-species distribution patterns. - Mar. Ecol. Progr. Ser. 8, 37-52.

Götting, K.-J., 1973. Die Polyplacophora der karibischen Küste Kolumbiens. - Arch. Molluskenk. $103,243-261$.

Gray, J. S., 1981. The ecology of marine sediments. Cambridge Univ. Press, Cambridge, 154 pp.

Hadfield, M. G., 1976. Molluscs associated with living tropical corals. - Micronesica 12, $133-148$.

Haeupler, H., 1982. Evenness als Ausdruck der Vielfalt in der Vegetation. Cramer, Vaduz, 268 pp.

Hemmen, J. \& Hemmen, C., 1979. Beitrag zur Kenntnis der Meeresmollusken-Fauna der Karibischen See. - Jb. nassau. Ver. Naturk. 104, 137-172.

Heybrock, G., 1984. Der Tayrona-Trockenwald Nord-Kolumbiens. - Giessener geogr. Schr. 23, $1-88$.

Humfrey, M., 1975. Sea shells of the West Indies. Collins, Glasgow, $351 \mathrm{pp}$.

Kaufmann, R. \& Götting, K.-J., 1970. Prosobranchia aus dem Litoral der karibischen Küste Kolumbiens. - Helgoländer wiss. Meeresunters. 21, 333-398.

Marcus, E. du B.-R., 1976. Opisthobranchia von Santa Marta, Colombia. - Stud. neotrop. Fauna 11, $119-150$.

Moore, D. R., 1972. Ecological and systematic notes on Caecidae from St. Croix, U. S. Virgin Islands. - Bull. mar. Sci. 22, 881-899.

Nowell-Usticke, G. W., 1959. A check list of the marine shells of St. Croix, U. S. Virgin Islands. Lane Press, Burlington, 90 pp.

Olsson, A. A. \& McGinty, T, L., 1958. Recent marine mollusks from the Caribbean coast of Panamá with the description of some new genera and species. - Bull. Am. Paleont. 39, 5-58.

Petersen, C. G. J., 1915. On the animal communities of the sea bottom in the Skagerrak, the Christiana Fjord and the Danish waters. - Rep. Dan. biol. Stn 23, 3-28.

Petuch, E. J., 1976. An unusual molluscan assemblage from Venezuela. - Veliger 18, 322-325.

Petuch, E. J, 1980. A reanalysis of neogene Caribbean provinciality with reference to the discovery of a relict coenogastropod fauna off northern South America. Diss., Univ. Miami, Coral Gables, $163 \mathrm{pp}$.

Petuch, E. J., 1981. A relict neogene coenogastropod fauna from northern South America. Malacologia 20, 307-347.

Princz, D., 1973. Los moluscos gastrópodos y pelecypodos del Estado de Nueva Esparta, Venezuela. - Contr. estad. Invest. mar. Margarita, Fund. La Salle Cienc. nat. 50, 169-222.

Princz, D., 1977. Notas sobre algunos micromoluscos de la plataforma de Guayana. - Mems Soc. Cienc. nat. La Salle 37, 283-293.

Princz, D., 1978. Los moluscos marinos del Golfo de Venezuela. - Contr. estad. Invest. mar. Margarita, Fund. La Salle Cienc. nat. 78, 51-76. 
Princz, D., 1982. Lista y bibliografía de los gastrópodos marinos vivos de los mares de Venezuela, Trinidad e Islas de Sotavento. - Boln Soc. venez. Cienc. nat. 37, 103-147.

Radwin, G. E., 1969. A recent molluscan fauna from the Caribbean coast of southeastern Panamá.Trans. San Diego Soc. nat. Hist. 15, 229-236.

Robertson, R, 1970. Review of the predators and parasites of stony corals, with special reference to symbiotic prosobranch gastropods. - Pacif. Sci. 24, 43-54.

Rodríguez, G., 1959. The marine communities of Margarita Island. - Bull. mar. Sci. Gulf Caribb. 9 , $237-280$.

Schnetter, R., 1968. Vegetation des Cerro San Fernando und des Cerro La Llorona im Trockengebiet bei Santa Marta, Kolumbien. - Ber. dt. bot. Ges. 81, 283-302.

Seshappa, G., 1953. Observations on the physical and biological features of the inshore sea bottom along the Malabar coast. - Proc. natn. Inst. Sci. India 19, 257-279.

Shannon, C. E., 1976. Die mathematische Theorie der Kommunikation. In: Mathematische Grundlagen der Informationstheorie. Hrsg. von C. E. Shannon \& W. Weaver. Oldenbourg, Wien, $41-143$.

Tello, J., 1975. Catálogo de la fauna venezuelana. VIII. Mollusca. Publ. Com. organiz. III. Conf. Nac. Unid. Derecho Mar. Caracas, 599 pp.

Thorson, G., 1971. Life in the sea. McGraw-Hill, New York, 256 pp.

Vokes, H. E. \& Vokes, E. H., 1983. Distribution of shallow-water marine Mollusca, Yucatán Peninsula, Mexico. - Publs middle Am. Res. Inst., Tulane Univ. 54, 1-183.

Wade, B. A., 1967. Studies on the biology of the West Indian clam, Donax denticulatus Linné. 1. Ecology, - Bull. mar. Sci. 17, 149-174.

Wedler, E., 1975. Okologische Untersuchungen an Hydroiden des Felslitorals von Santa Marta (Kolumbien). - Helgoländer wiss. Meeresunters. 27, 324-363.

Werding, B. \& Erhardt, H., 1977. Un encuentro de Madracis myriaster (Milne-Edwards y Haime) (Scleractinia) en la Bahía de Santa Marta, Colombia. - An. Inst. Invest. mar., Punta Betín 9, 105-107.

Wilhelmy, H., 1954. Die klimatologische und pflanzengeographische Entwicklung des Trockengebietes am Nordrand Südamerikas seit dem Pliozän. - Erde 6, 244-273. 